\title{
Gate-Voltage Response of a One-Dimensional Ballistic Spin Valve without Spin-Orbit Interaction
}

\author{
Maciej Misiorny ${ }^{1,2, *}$ and Carola Meyer ${ }^{3,4}$ \\ ${ }^{1}$ Department of Microtechnology and Nanoscience MC2, \\ Chalmers University of Technology, SE-412 96 Göteborg, Sweden \\ ${ }^{2}$ Faculty of Physics, Adam Mickiewicz University, 61-614 Poznań, Poland \\ ${ }^{3}$ Fachbereich Physik, Universität Osnabrück, D-49069 Osnabrück, Germany \\ ${ }^{4}$ Peter Grünberg Institut (PGI-6), Forschungszentrum Jülich and JARA-Fundamentals of Future \\ Information Technologies, DE-52 425 Jülich, Germany
}

(Received 17 June 2016; revised manuscript received 21 December 2016; published 9 February 2017)

\begin{abstract}
We show that the engineering of tunnel barriers forming at the interfaces of a one-dimensional spin valve provides a viable path to a strong gate-voltage tunability of the magnetoresistance effect. Specifically, we investigate theoretically a carbon nanotube (CNT) spin valve in terms of the influence of the CNT-contact interface on the performance of the device. The focus is on the strength and the spin selectivity of the tunnel barriers that are modeled as Dirac- $\delta$ potentials. The scattering matrix approach is used to derive the transmission coefficient that yields the tunneling magnetoresistance (TMR). We find a strong nontrivial gate-voltage response of the TMR in the absence of spin-orbit coupling when the energy of the incident electrons matches the potential energy of the barrier. Analytic expressions for the TMR in various limiting cases are derived. These expressions are used to explain previous experimental results, and also to predict prospective ways for device optimization with respect to the size and tunability of the TMR effect in the ballistic transport regime by means of engineering the tunnel barriers at the CNT-contact interfaces.
\end{abstract}

DOI: 10.1103/PhysRevApplied.7.024011

\section{INTRODUCTION}

The already-vast and still-growing research area of spintronics offers an alternative functionality for solid-state devices based on the spin of the electrons rather than their charge $[1,2]$. In this context, graphene and carbon nanotubes (CNTs) are regarded as extremely promising materials [3-5] since the spin lifetime is long due to the small spin-orbit coupling and due to a low natural abundance of $\mathrm{C}^{13}$ nuclear spins [6-10]. Additionally, the Fermi velocity of these materials is very high, resulting in short dwell times within a device. This combination can, in turn, lead to a large magnetoresistance (MR) effect as well as to a large absolute change of resistance-both important for a good performance of spin-valve devices [4]. A key issue for such devices is to enhance the spin-injection efficiency by optimizing both the contact material [3] and the tunnel barrier $[4,10,11]$.

Another major point, not yet discussed in depth, regards the fact that many interesting effects in spintronic devices stem from the spin-orbit coupling $[12,13]$. To tune the MR effect efficiently with a gate voltage, for instance, as in a spin transistor, a strong spin-orbit coupling (SOC) is desired. Though the SOC is very weak in graphene [14], it can be strongly enhanced by adatoms that induce local $s p^{3}$ hybridization in the carbon bonds [15]. On the

*misiorny@amu.edu.pl other hand, the spin-orbit interaction in CNTs is stronger, for the same reason, due to their curvature [16,17] and has been found to be even larger in some devices [18]. A gate voltage will tune the MR effect in such materials rather efficiently; however, this tuning is usually achieved at the expense of the spin relaxation time, which is the great asset of carbon materials.

In this paper, we present model calculations that reveal another option for gate-controlled spin devices that avoids enhancing the spin-orbit coupling and, therefore, the spin relaxation. We demonstrate that, in quasi-one-dimensional devices, the MR effect can show a strong tunability with gate voltage, depending on the properties of tunnel barriers arising at the interfaces of the electrodes. Specifically, we systematically analyze how the strength and spin selectiveness of these tunnel barriers affect magnetotransport characteristics of a ballistic onedimensional spin valve employing a CNT as model system. This aspect seems to be of key importance for a full understanding of the injection of spin-polarized electrons into a CNT, and it has not been examined in full detail hitherto. Actually, although spin-dependent transport through a CNT attached to ferromagnetic metallic electrodes has been the subject of extensive experimental studies for almost two decades [19-24], only recently has the role of the tunnel-barrier strength in this process been addressed [10] - showing that the MR of a device is significantly influenced by this factor. 
For the purpose of this study, we consider a CNT-based spin valve that basically acts as an electronic interferometer, a setup employed formerly both in experiments [25-27] and theoretically $[28,29]$. In order to capture the effect of electrode-CNT interfaces, at which tunnel barriers form, we treat them as spin-dependent Dirac- $\delta$ potentials. A similar approach has been used to study spin injection between a ferromagnetic metal and a two-dimensional electron system [30,31]. Then, calculations of spin-dependent transport through a device are derived by means of the scatteringmatrix approach. We find that the engineering of the strength and spin-selective properties of the tunnel barriers, in combination with the gate-voltage tuning, provides a path for obtaining devices in which the magnitude of the MR effect can be adjusted in a broad range extending from $-15.5 \%$ up to $+40 \%$.

The paper is organized as follows. First, in Sec. II, we introduce the model of a CNT-based spin valve and define the concept of a tunnel barrier at the electrode-CNT interface. Next, in Sec. III, we provide a theoretical description of spin injection through the interface and derive the corresponding transmission coefficient for conduction electrons. This derivation enables us to determine the linear transport through the device, as outlined in Sec. IV. Numerical results are presented in Sec. V, where we discuss cases of both single (Sec. VA) and many orbital (Sec. V B) transport channels. In that section, we also consider in detail the limit of strong tunnel barriers (Sec. VA 1), as well as the situation where a device is characterized by the asymmetric (Sec. VA 2) and spin-selective (Sec. VA 3) barriers. Finally, we conclude the paper in Sec. VI with a discussion about possible implementations of such barriers regarding the essential effects and the general performance to be expected from prospective devices.

\section{MODEL OF A CNT-BASED SPIN VALVE}

A device under consideration consists of two ferromagnetic (FM) metallic leads interconnected by a CNT which we approximate as a ballistic and noninteracting onedimensional (1D) quantum wire [29,32,33]; see Fig. 1(a). Importantly, at both CNT-lead interfaces, a tunnel barrier can form whose exact shape, generally different for each interface, is unknown. For this reason, we model scattering of tunneling electrons at the interfaces by means of a spinselective repulsive Dirac- $\delta$ potential $U_{\sigma}^{q} \delta\left(z_{q}\right)$ for $q=L$ (left), $R$ (right); see Fig. 1(b). Such an approach has already been shown to suffice in capturing key transport features of the interface $[30,31,34,35]$, but it has not yet been systematically applied to analyze how its properties affect one-dimensional spin transport.

In the model to be analyzed, two identical FM leads are described as a reservoir of noninteracting, itinerant electrons within the Stoner model, with the dispersion relation given by

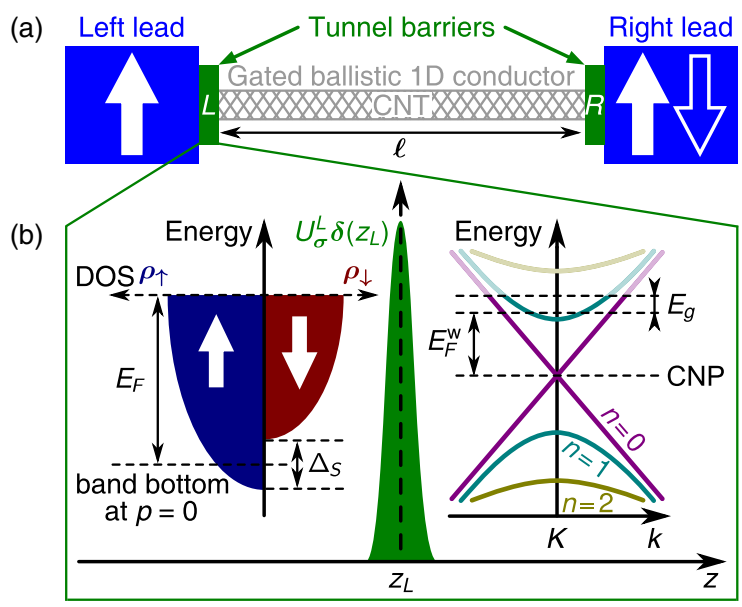

FIG. 1. (a) A graphic depiction of the model device: a gated CNT inserted between two ferromagnetic (FM) metallic leads whose spin moments are oriented either parallel or antiparallel. (b) Schematic representation of a tunnel barrier arising at the left CNT-lead interface that is modeled as a spin-selective repulsive Dirac- $\delta$ potential $U_{\sigma}^{L} \delta\left(z_{L}\right)$. In the left (right) side of (b), a sample dispersion relation for a FM lead (a CNT around the Fermi point $K$ ) is shown. Here, CNP stands for the charge-neutrality point with respect to which energy is measured. For a detailed description, see Sec. II.

$$
\varepsilon_{\sigma}=\frac{\hbar^{2}\left(k_{\sigma}\right)^{2}}{2 m^{*}}-\eta_{\sigma} \frac{\Delta_{S}}{2}-E_{F} .
$$

Here, $\Delta_{S}$ denotes the Stoner splitting, $\eta_{\uparrow(\downarrow)}= \pm 1$, and $E_{F}$ represents the Fermi energy-note that the energy $\varepsilon_{\sigma}$ is measured relative to the Fermi level. Additionally, we assume the effective mass to be equal to the electron's mass, $m^{*} \approx m_{e}$. Generally, in a bulk system with a parabolic dispersion (i.e., for the free-electron model) the spin-dependent density of states (DOS) $\rho_{\sigma}$ at the Fermi level (per unit volume and per spin channel) is related to the spin-dependent Fermi wave vector, $k_{F \sigma} \equiv k_{\sigma}\left(\varepsilon_{\sigma}=0\right)$, as $\rho_{\sigma}=2 m_{e} k_{F \sigma} /\left(4 \pi^{2} \hbar^{2}\right)$, as shown on the left side of Fig. 1(b). With such a definition of DOS, we introduce the spin-polarization coefficient $p$ for the material of which leads are made [36],

$$
p=\frac{\rho_{+}-\rho_{-}}{\rho_{+}+\rho_{-}},
$$

with the spin index $\eta= \pm$ referring now to spin-majority $(+)$ and spin-minority (-) electrons. Note that the notion of spin-majority and -minority electrons is useful in the present case because two different collinear configurations of spin moments of electrodes-that is, parallel $(\mathrm{P})$ and antiparallel (AP)—will be considered. Specifically, the orientation of a spin moment of the left electrode will be kept fixed so that the relation between "spin-up" and "spindown" electrons and spin-majority and -minority electrons in the left electrode takes the following form: 


$$
\mathrm{P} / \mathrm{AP}\left(\sigma_{L}\right) \equiv \begin{cases}+ \text { if } & \sigma=\uparrow \\ - \text { if } & \sigma=\downarrow\end{cases}
$$

This fixed orientation of a spin moment in the left electrode also sets the reference frame for spin orientations of electronic spins in the right electrode. As a result, when a spin moment of the right electrode is parallel or antiparallel with respect to the left one, we get, respectively,

$$
\mathrm{P}\left(\sigma_{R}\right) \equiv \begin{cases}+ \text { if } & \sigma=\uparrow, \\ - \text { if } & \sigma=\downarrow\end{cases}
$$

and

$$
\operatorname{AP}\left(\sigma_{R}\right) \equiv \begin{cases}+ \text { if } & \sigma=\downarrow \\ - \text { if } & \sigma=\uparrow .\end{cases}
$$

Moreover, in the limit of moderate spin polarizations observed in typical materials used for electrodes [36,37] $\Delta_{S} /\left(2 E_{F}\right)<1$, so that a wave vector can be approximated as $k_{F \eta} \approx k_{0}\left[1+\eta \Delta_{S} /\left(4 E_{F}\right)\right]$, with $k_{0}=\sqrt{2 m_{e} E_{F}} / \hbar$, and, consequently, one can use the following parametrization of the Stoner splitting parameter $\Delta_{S}=4 E_{F} p$. Note that the above approximation remains valid only for moderate values of the spin polarization of the electrodes $(p<0.5)$.

Next, essential features of a CNT in the vicinity of the Fermi point $K\left(K^{\prime}\right)$ are captured by a dispersion relation $[32,33,38]$,

$$
\varepsilon_{n}^{w}= \pm \hbar v_{F}^{w} \sqrt{\left(k_{n}^{w}-k_{F}^{w}\right)^{2}+(n / r)^{2}}+E_{g}-E_{F}^{w},
$$

typical for 1D conductors [39], with the $+(-)$ sign corresponding to the conduction (valence) band; see the right side of Fig. 1(b). In Eq. (6), $v_{F}^{w}$ stands for the Fermi velocity and $n / r$ represents the quantized transverse momentum of a metallic CNT [38], with $r$ denoting the radius of a CNT and $n=0,1,2, \ldots$ being the subband index. As above, the energy $\varepsilon_{n}^{w}$ is defined relative to the Fermi level $E_{F}^{w}$. Recall that, for an undoped CNT, it coincides with the charge-neutrality point (CNP), i.e., $E_{F}^{w}=0$, so that only one orbital channel $(n=0)$ can contribute to transport at low temperature. However, owing to modification of the immediate environment of a CNT, $E_{F}^{w}$ can be shifted by as much as $\pm 1 \mathrm{eV}[40,41]$, and more channels thus become available for transport. The Fermi level can be further adjusted by application of an external gate voltage, which leads to the shift $E_{g}$ due to the capacitive coupling between the gate and a CNT [40]. Note that Eq. (6) remains valid as long as the variation in $E_{g}$ is small; that is, the Fermi level is moderately shifted around $E_{F}^{w}$. It is assumed that transport of electrons along a CNT is ballistic and no mixing of channels occurs.

Finally, before we turn to the discussion of electron tunneling through the electrode-CNT interface, we would like to briefly comment on applicability limits of the model under consideration. We recall that electrodes are here approximated by only free ( $s$-band) electrons, and tunnel barriers are treated as a Dirac- $\delta$ potential. In fact, the tunnel barrier forming at the electrode-CNT interface can be of a much more complex nature, with a potential profile determined by additional factors not included in the present considerations, like the interface roughness and adsorbates $[42,43]$. Furthermore, materials typically used for electrodes involve transition metals and their alloys, in the case of which the free-electron model may be insufficient to capture all key features. For example, for these materials, a more complicated band structure is expected to underlie the tunneling of electrons across the interface [44]. In order to accommodate fully all of these intricacies-that is, the complex electrode-CNT hybridization and the exact morphology of the interface- a model from first principles is needed $[45,46]$. Nevertheless, the present approach already shows great potential for one-dimensional CNT spin valves in the ballistic transport regime with respect to the size and tunability of the MR effect.

\section{TUNNELING THROUGH A FM-METAL-CNT INTERFACE}

Spin injection across an interface with the band structure mismatch at the Fermi energy has already been addressed, e.g., for FM-metal-metal [47] and FM-metalsemiconductor heterojunctions [30,31]. Here, we consider a spin-dependent tunneling of electrons through the FMmetal-CNT interface, as illustrated in Fig. 1(b). The relevant transmission coefficient $\mathcal{T}$ can be derived by means of standard quantum-mechanical methods. The key problem one has to face is then how to match the wave functions at the interface. Let us focus on the left interface for the moment.

For an ideal interface (i.e., without spin-flip and inelastic or interchannel scattering) the particle current $j_{\sigma n}^{z}$ along the $z$ axis across the interface has to be conserved in each spin $(\sigma)$ and orbital $(n)$ channel. This requirement basically means that the current in the vicinity of the barrier on its left side, $j_{\sigma n}^{z}\left(z_{L}^{-}\right)$, has to match that on the right side, $j_{\sigma n}^{z}\left(z_{L}^{+}\right)$, namely, $j_{\sigma n}^{z}\left(z_{L}^{-}\right)=j_{\sigma n}^{z}\left(z_{L}^{+}\right)$, with $z_{L}^{ \pm} \equiv z_{L} \pm 0^{+}$and $0^{+}$ denoting an infinitesimally small displacement. Close to the interface, on its left side $\left(z<z_{L}\right)$, corresponding to a FM metal, this current is given by

$$
\begin{aligned}
& j_{\sigma n}^{z}\left(z<z_{L}\right) \\
& \quad=\frac{i \hbar}{2 m_{e}}\left\{\left[\partial_{z} \Psi_{\sigma n}^{\dagger}(z)\right] \underline{\boldsymbol{\sigma}}^{0} \boldsymbol{\Psi}_{\sigma n}(z)-\boldsymbol{\Psi}_{\sigma n}^{\dagger}(z) \underline{\boldsymbol{\sigma}}^{0}\left[\partial_{z} \boldsymbol{\Psi}_{\sigma n}(z)\right]\right\},
\end{aligned}
$$

whereas on the right side $\left(z>z_{L}\right)$-that is, in a CNT-it takes the form 


$$
j_{\sigma n}^{z}\left(z>z_{L}\right)=v_{F}^{w} \boldsymbol{\Phi}_{\sigma n}^{\dagger}(z) \underline{\boldsymbol{\sigma}}^{z} \boldsymbol{\Phi}_{\sigma n}(z),
$$

with $\underline{\sigma}^{z}\left(\underline{\sigma}^{0}\right)$ denoting the Pauli (identity) matrix, and the wave functions $\boldsymbol{\Psi}$ and $\boldsymbol{\Phi}$ defined as

$$
\Psi_{\sigma n}(z)=\left(\begin{array}{c}
\psi_{\sigma n}^{\vec{\sigma}} e^{i k_{\sigma} z} \\
\psi_{\sigma n}^{\leftarrow} e^{-i k_{\sigma} z}
\end{array}\right)
$$

and

$$
\boldsymbol{\Phi}_{\sigma n}(z)=\left(\begin{array}{c}
\phi_{\sigma n}^{\overrightarrow{\sigma n}} e^{i k_{n}^{w} z} \\
\phi_{\sigma n}^{\leftarrow} e^{-i k_{n}^{w} z}
\end{array}\right)
$$

Here, $\psi_{\sigma n}^{d}$ and $\phi_{\sigma n}^{d}$ generally represent the respective probability amplitude for right- $(d=\rightarrow)$ and left- $(d=\leftarrow)$ moving electrons. Inserting Eqs. (9) and (10) into the expressions for $j_{\sigma n}^{z}(z)$, one obtains $j_{\sigma n}^{z}\left(z<z_{L}\right)=$ $\left(\hbar k_{\sigma} / m_{e}\right)\left[\left|\psi_{\sigma n}\right|^{2}-\left|\psi_{\sigma n}^{\leftarrow}\right|^{2}\right]$ and $j_{\sigma n}^{z}\left(z>z_{L}\right)=v_{F}^{w}\left[\left|\phi_{\sigma n}\right|^{2}-\right.$ $\left.\left|\phi_{\sigma n}^{\leftarrow}\right|^{2}\right]$. Consequently, one can define the transmission amplitude for electrons incident on the left interface from the left $(\rightarrow)$ or right $(\leftarrow)$ in terms of the flux amplitude as

$$
\mathcal{T}_{\sigma n}^{L \rightarrow}=\left|\frac{\sqrt{v_{F}^{w}} \phi_{\sigma n}^{\vec{\sigma}}}{\sqrt{\hbar k_{\sigma} / m_{e}} \psi_{\sigma n}^{\overrightarrow{\sigma n}}}\right|^{2}
$$

and

$$
\mathcal{T}_{\sigma n}^{L \leftarrow}=\left|\frac{\sqrt{\hbar k_{\sigma} / m_{e}} \psi_{\sigma n}^{\leftarrow}}{\sqrt{v_{F}^{w}} \phi_{\sigma n}^{\leftarrow}}\right|^{2}
$$

Analogous definitions also hold for the right interface. Interestingly, one can note that the same result for $j_{\sigma n}^{z}\left(z>z_{L}\right)$ can be reached if one uses the free-electron model, for which

$$
\begin{aligned}
& j_{\sigma n}^{z}\left(z>z_{L}\right) \\
& \quad=\frac{i \hbar}{2 m_{n}^{*}}\left\{\left[\partial_{z} \boldsymbol{\Phi}_{\sigma n}^{\dagger}(z)\right] \underline{\boldsymbol{\sigma}}^{0} \boldsymbol{\Phi}_{\sigma n}(z)-\boldsymbol{\Phi}_{\sigma n}^{\dagger}(z) \underline{\boldsymbol{\sigma}}^{0}\left[\partial_{z} \boldsymbol{\Phi}_{\sigma n}(z)\right]\right\},
\end{aligned}
$$

with the effective mass $m_{n}^{*}=\hbar k_{n}^{w} / v_{F}^{w}$. For this reason, the continuity of the current across the $q$ th interface between a FM lead and a CNT can be ensured by imposing the following boundary conditions for wave functions [48-50]:

$$
\begin{aligned}
& \sqrt{\frac{M_{n}}{m_{e}}} \operatorname{Tr}\left[\underline{\boldsymbol{\sigma}}^{0} \boldsymbol{\Psi}_{\sigma n}\left(z_{q}\right)\right]=\sqrt{\frac{M_{n}}{m_{n}^{*}}} \operatorname{Tr}\left[\underline{\boldsymbol{\sigma}}^{0} \boldsymbol{\Phi}_{\sigma n}\left(z_{q}\right)\right], \\
& \sqrt{\frac{M_{n}}{m_{n}^{*}}} \operatorname{Tr}\left[\left.\underline{\boldsymbol{\sigma}}^{0} \partial_{z} \boldsymbol{\Phi}_{\sigma n}(z)\right|_{z_{q}}\right]-\sqrt{\frac{M_{n}}{m_{e}}} \operatorname{Tr}\left[\left.\underline{\sigma}^{0} \partial_{z} \boldsymbol{\Psi}_{\sigma n}(z)\right|_{z_{q}}\right] \\
& =\frac{2 M_{n} U_{\sigma}^{q}}{\hbar^{2}} \sqrt{\frac{M_{n}}{m_{n}^{*}}} \operatorname{Tr}\left[\underline{\boldsymbol{\sigma}}^{0} \boldsymbol{\Phi}_{\sigma n}\left(z_{q}\right)\right],
\end{aligned}
$$

with $M_{n}=\sqrt{m_{e} m_{n}^{*}}$. The transmission coefficient $\mathcal{T}_{\sigma n}^{q} \equiv$ $\mathcal{T}_{\sigma n}^{q \rightarrow}=\mathcal{T}_{\sigma n}^{q \leftarrow}$, which characterizes the tunneling of an electron with spin $\sigma$ to or out the $n$th channel of a CNT across the $q$ th interface, takes thus the following form $\left(\varepsilon=\varepsilon_{\sigma}=\varepsilon_{n}^{w}\right)$ :

$$
\mathcal{T}_{\sigma n}^{q c}(\varepsilon)=\frac{4 k_{c\left(\sigma_{q}\right)}(\varepsilon) k_{n}^{w}(\varepsilon)}{\left[k_{c\left(\sigma_{q}\right)}(\varepsilon)+k_{n}^{w}(\varepsilon)\right]^{2}+\left[Z_{\sigma}^{q}\right]^{2} k_{n}^{w}(\varepsilon) \kappa} .
$$

The action of the magnetic configuration index $c\left(\sigma_{q}\right)$, which, for a given configuration $c=\mathrm{P}$, AP, relates spin- $\sigma_{q}$ electrons to spin-majority or -minority electrons in the $q$ th electrode, should be interpreted by means of Eqs. (3)-(5). Furthermore, $\kappa=2 E_{F} /\left(\hbar v_{F}^{w}\right)$, and $Z_{\sigma}^{q}=k_{0} U_{\sigma}^{q} / E_{F}$ is the spin-selective dimensionless barrier strength, defined as the ratio of the spin-dependent potential energy of the barrier $k_{0} U_{\sigma}^{q}$ to the energy of an incident electron from the Fermi level of a lead. Here, we additionally introduce the spin-asymmetry parameter $\alpha_{q}$ for the $q$ th barrier,

$$
\alpha_{q}=\frac{Z_{\uparrow}^{q}-Z_{\downarrow}^{q}}{Z_{\uparrow}^{q}+Z_{\downarrow}^{q}},
$$

so that $Z_{\sigma}^{q}=Z_{q}\left(1+\eta_{\sigma} \alpha_{q}\right)$ and $Z_{q}=\left(Z_{\uparrow}^{q}+Z_{\downarrow}^{q}\right) / 2$, with $-1<\alpha_{q}<1$. Note that a positive (negative) $\alpha_{q}$ means that the probability for spin-down (spin-up) electrons to tunnel through the barrier is higher due to a smaller barrier strength. The limit of $\alpha_{q} \rightarrow+1(-1)$ corresponds, then, to a vanishingly small barrier, i.e., almost perfect transmission, for spin-down (spin-up) electrons. Importantly, the spin selectiveness of a tunnel barrier, characterized by the parameter $\alpha_{q}$, is an inherent property of the barrier and is not associated with the magnetic configuration of the electrodes. For example, note that, in Eq. (16), the magnetic-configuration index $c\left(\sigma_{q}\right)$ affects only the wave vectors of electrons in the electrode. This effect should not be confused with the spin dependence of the transmission coefficient $\mathcal{T}_{\sigma n}^{q c}(\varepsilon)$ of a barrier, which involves both the effects of the barrier spin selectiveness (determined by the spin asymmetry $\alpha_{q}$ ) and the magnetic properties of the electrodes (characterized both by the spin polarization $p$ and the magnetic configuration of the valve).

\section{LINEAR TRANSPORT THROUGH A CNT-BASED SPIN VALVE}

Within the scattering-matrix approach, the linearresponse conductance at temperature $T$ is given by [51]

$$
G_{\mathrm{P} / \mathrm{AP}}=\left(\frac{e^{2}}{h}\right) \frac{1}{4 k_{B} T} \sum_{n \sigma} \int d \varepsilon \mathscr{T}_{\sigma n}^{\mathrm{P} / \mathrm{AP}}(\varepsilon) \cosh ^{-2}\left(\frac{\varepsilon}{k_{B} T}\right),
$$

where $\mathscr{T}_{n \sigma}^{\mathrm{P} / \mathrm{AP}}(\varepsilon)$ stands for the transmission coefficient of an electron with spin $\sigma$ passing through the $n$th channel of a device in the P or AP magnetic configuration [28,51], 


$$
\mathscr{T}_{\sigma n}^{c}(\varepsilon)=\frac{\mathcal{T}_{\sigma n}^{L c}(\varepsilon) \mathcal{T}_{\sigma n}^{R c}(\varepsilon)}{\left|1-\sqrt{\left[1-\mathcal{T}_{\sigma n}^{L c}(\varepsilon)\right]\left[1-\mathcal{T}_{\sigma n}^{R c}(\varepsilon)\right]} \mathrm{e}^{i \theta_{\sigma n}^{c}(\varepsilon)}\right|^{2}} .
$$

In the equation above, $\theta_{\sigma n}^{c}(\varepsilon)=2 \delta_{n}(\varepsilon)+\varphi_{\sigma n}^{L c}(\varepsilon)+\varphi_{\sigma n}^{R c}(\varepsilon)$ is the quantum-mechanical phase an electron acquires during its resonant transport through a CNT. Here, the first term, $\delta_{n}(\varepsilon)=\ell\left[k_{F}^{w}+\sqrt{\left(\varepsilon+E_{F}^{w}-E_{g}\right)^{2} /\left(\hbar v_{F}^{w}\right)^{2}-(n / r)^{2}}\right]$ [cf. Eq. (6)], corresponds to the phase stemming from the ballistic propagation of an electron between the opposite interfaces of a CNT of the length $\ell$, while the second one, $\varphi_{\sigma n}^{L c}(\varepsilon)+\varphi_{\sigma n}^{R c}(\varepsilon)$, represents the spin-dependent interfacial phase shift [28] that arises when an electron is scattered at the left $(L)$ and right $(R)$ interface back into a CNT. This shift is basically related to the reflection amplitudes as $\varphi_{\sigma n}^{L}(\varepsilon)=\arg \left[r_{\sigma n}^{L \leftarrow}(\varepsilon)\right]$ and $\varphi_{\sigma n}^{R}(\varepsilon)=\arg \left[r_{\sigma n}^{R \rightarrow}(\varepsilon)\right]$, with the amplitudes at the interfaces defined as $r_{\sigma n}^{L \leftarrow}=\phi_{\sigma n} / \phi_{\sigma n}^{\leftarrow}$ and $r_{\sigma n}^{R \rightarrow}=\phi_{\sigma n}^{\leftarrow} / \phi_{\sigma n}$, so that one finds

$\varphi_{\sigma n}^{q c}(\varepsilon)=\arg \left(\frac{-k_{c\left(\sigma_{q}\right)}(\varepsilon)+k_{n}^{w}(\varepsilon)-i Z_{\sigma}^{q} \sqrt{k_{n}^{w}(\varepsilon) \kappa}}{k_{c\left(\sigma_{q}\right)}(\varepsilon)+k_{n}^{w}(\varepsilon)+i Z_{\sigma}^{q} \sqrt{k_{n}^{w}(\varepsilon) \kappa}}\right)$.

Finally, one can note that, in the limit of low temperature, only electrons from the vicinity of the Fermi level $(\varepsilon=0)$ that is, from the energy window of a few $k_{B} T$ around the Fermi level—contribute to transport; cf. Eq. (18). At such an energy scale, wave vectors $k_{\sigma}(\varepsilon)$ and $k_{n}^{w}(\varepsilon)$ vary insignificantly and, as a consequence, the change of $\mathcal{T}_{\sigma n}^{q}(\varepsilon)$ and $\varphi_{\sigma n}^{q}(\varepsilon)$ with the energy is negligibly small. Therefore, in the following discussion, we assume $\mathcal{T}_{\sigma n}^{q c}(\varepsilon) \approx \mathcal{T}_{\sigma n}^{q c}(0) \equiv \mathcal{T}_{\sigma n}^{q c}$ and $\varphi_{\sigma n}^{q c}(\varepsilon) \approx \varphi_{\sigma n}^{q c}(0) \equiv \varphi_{\sigma n}^{q c}$.

\section{NUMERICAL RESULTS AND DISCUSSION}

In order to discuss the dependence of spin-dependent transport through a CNT-based spin valve on the strength and properties of tunneling barriers at the interfaces, we consider a model CNT of the length $\ell=100 \mathrm{~nm}$ and the radius $r=2 \mathrm{~nm}$, characterized by $v_{F}^{w}=8 \times 10^{6} \mathrm{~m} / \mathrm{s}$ and $k_{F}^{w}=8.5 \mathrm{~nm}^{-1}$ [25]. As a result, the spacing between the subbands at the Fermi point amounts to $\Delta E \approx 260 \mathrm{meV}$. Furthermore, we assume that electrodes are described by the Fermi energy $E_{F}=8.5 \mathrm{eV}$ and the spin-polarization parameter $p=0.25$. Such a value of $p$ is very realistic since common contact materials for CNTs, such as permalloy and CoPd, exhibit this degree of spin-polarized injection of electrons [10,52].

The change of transport properties of a spintronic device when switching between the parallel and antiparallel magnetic configuration is generally captured by the tunneling magnetoresistance (TMR),

$$
\mathrm{TMR}=\frac{G_{\mathrm{P}}-G_{\mathrm{AP}}}{G_{\mathrm{AP}}} .
$$

If the TMR is positive (negative), it basically means that conductance of the device is higher in the parallel (antiparallel) magnetic configuration than in the antiparallel (parallel) one.

In order to gain better insight into the expected effects, first, in Sec. VA, we consider the simplest conceptual case, that is, one with only one orbital channel $(n=0)$ available for transport. Such a case remains physically valid as long as the Fermi level of a CNT lies in the vicinity of the charge-neutrality point, $\left|E_{F}^{w}\right| \ll \Delta E$, so that, at low temperatures, the contribution of orbital channels (subbands) with $n \neq 0$ to transport can be neglected; see the right side of Fig. 1(b). Later on, in Sec. V B, we abandon this constraint and also discuss the case of many orbital channels by assuming that the Fermi level is shifted away from the charge-neutrality point.

\section{A. The case of a single orbital channel}

The hallmark of the model under discussion is the presence of the interference pattern in transport characteristics, as one can see in Fig. 2, where the TMR and conductance are plotted for a device with two identical tunnel barriers $\left(Z_{\sigma}^{L}=Z_{\sigma}^{R}=Z\right)$. It is clear that such a pattern in TMR stems directly from the periodic behavior of conductance as a function of the shift of the Fermi level due to a gate voltage $E_{g}$; see Figs. 2(d) and 2(f). Since the conductance of the device $G$, Eq. (18), is essentially determined by its transmission coefficient $\mathcal{T}$, Eq. (19), one can analyze $\mathcal{T}$ to obtain some basic information about the nature of such oscillations.

From Eq. (19), one immediately finds that, for a given $Z$, the transmission coefficient reaches its maximal achievable value at resonant energies for $p \in \mathbb{Z}$,

$$
\tilde{\varepsilon}_{\sigma p}^{c}=\Delta \varepsilon_{r}\left[p-\frac{1}{2 \pi}\left(\varphi_{\sigma}^{L c}+\varphi_{\sigma}^{R c}\right)\right]-\hbar v_{F}^{w} k_{F}^{w}+E_{g},
$$

with $\Delta \varepsilon_{r}=\pi \hbar v_{F}^{w} / \ell$ denoting the distance between consecutive resonances. Recall that here $E_{F}^{w}=0$, which basically means that only one orbital channel $(n=0)$ is active in transport. Thus, for the sake of notational clarity, in the remaining part of the present section, we omit the orbital channel (subband) index $n$. Importantly, one should notice that the position of these resonant states with respect to the Fermi level can be adjusted by application of a gate voltage, contributing via $E_{g}$. As a result, whenever $\tilde{\varepsilon}_{\sigma p}^{c}=0$, one observes resonant tunneling of an electron through a device, which manifests as increased conductance, as shown in Figs. 2(d) and 2(f). Moreover, it should be emphasized that $\tilde{\varepsilon}_{\sigma p}^{c}$ depends indirectly also on the strength of tunnel barriers $Z$ via the spin-dependent interfacial phase shifts $\varphi_{\sigma}^{q}$ [see Fig. 3(b)] and, consequently, also on the magnetic configuration of the electrodes. This effect is especially observable in the nontrivial behavior of the TMR for small values of $Z$; see, e.g., the long-dashed line for 

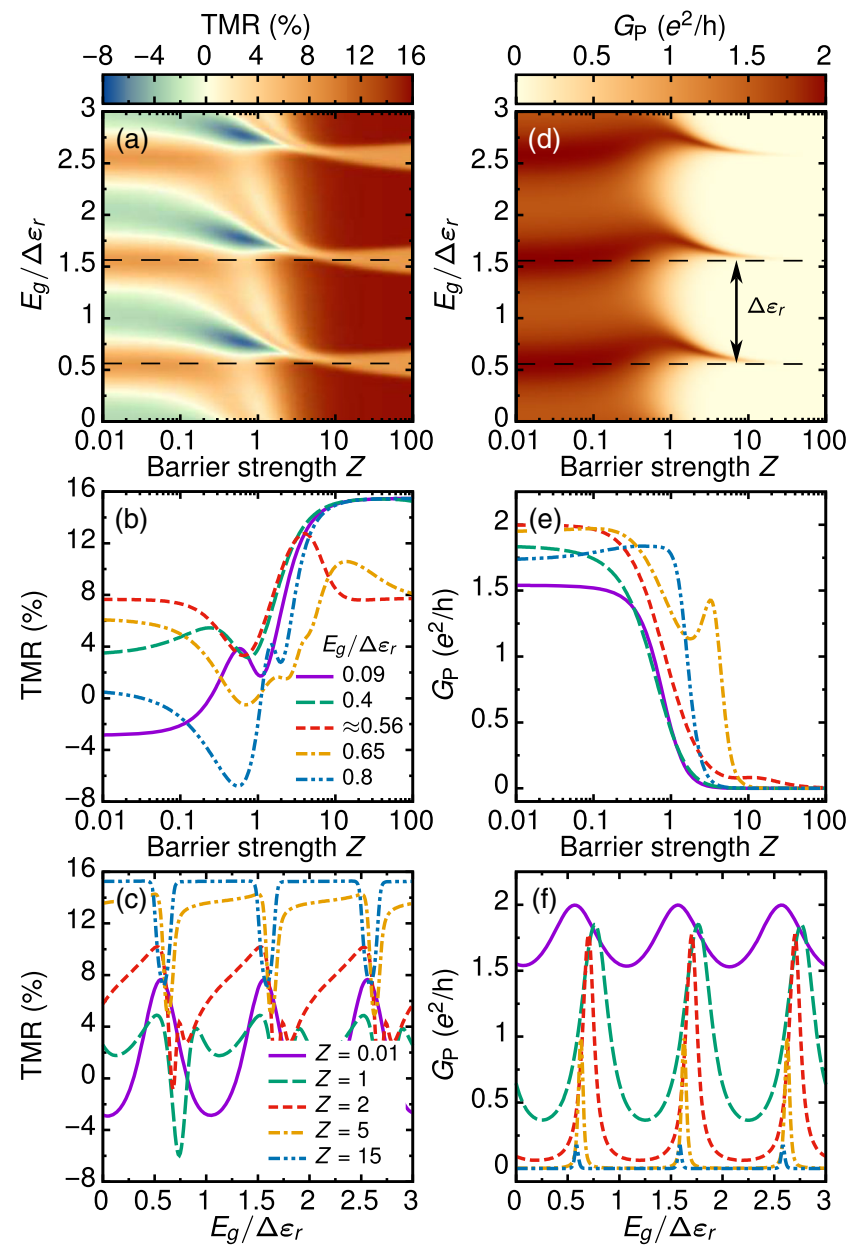

FIG. 2. Density maps of (a) tunneling magnetoresistance (TMR) and (d) conductance $G_{\mathrm{P}}$ in the parallel magnetic configuration shown as functions of the barrier strength $Z$ and the shift of the Fermi level due to a gate voltage $E_{g}$ in the case of two identical tunnel barriers at $T=2 \mathrm{~K}$. Here, $\Delta \varepsilon_{r}=$ $\pi \hbar v_{F}^{w} / \ell(\approx 16.5 \mathrm{meV})$ stands for the period of oscillations. Horizontal thin dashed lines indicate two sample values, $E_{g} / \Delta \varepsilon_{r} \approx 0.56$ and 1.56 , at which resonant dips in TMR occur for a large $Z$. The middle panels, (b) and (e), display the cross sections of (a) and (d), respectively, for selected values of $E_{g} / \Delta \varepsilon_{r}$ [see the legend in (b)], whereas the bottom panels, (c) and (f), are analogous cross sections, but now ones for chosen values of $Z$ [see the legend in (c)]. The other parameters are as specified in the main text.

$Z=1$ in Fig. 2(c), where the energy of an incident electron matches the potential energy of the barrier. In the opposite limit of a large $Z$, on the other hand, only sharp resonant dips in the TMR can be observed; see the double-dotteddashed line for $Z=15$ in Fig. 2(c). Note that, for small barriers where $Z=1$, the TMR can be tuned between $-8 \%$ and 4\%; see the long-dashed line in Fig. 2(c). These values are rather large considering the high conductance in this regime compared to the results in Man et al. [24]. It is, therefore, important, while fabricating devices, to keep in
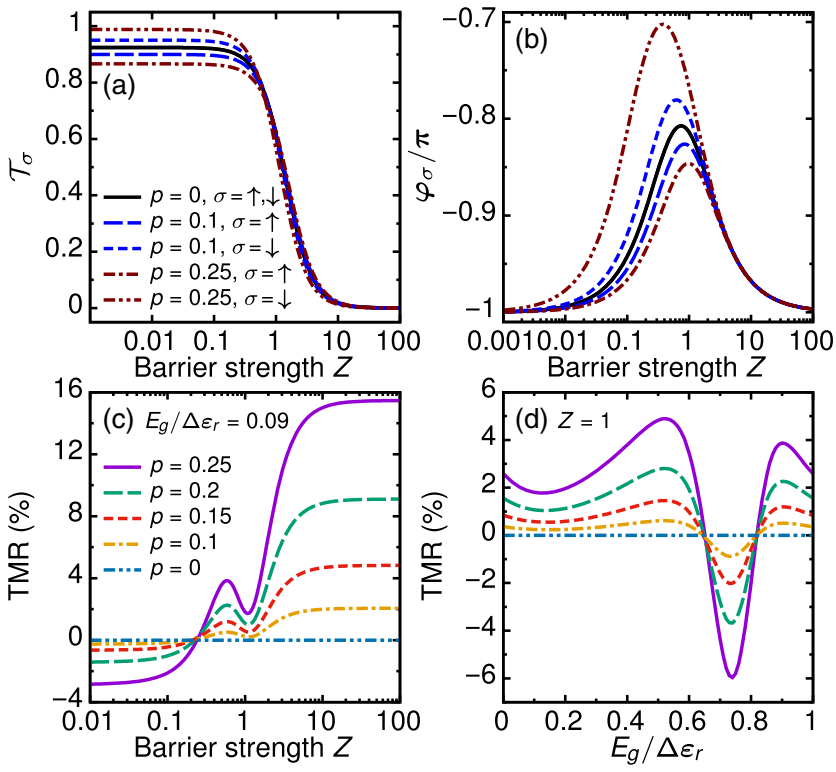

FIG. 3. The effect of spin-polarized electrodes, quantified by the spin-polarization coefficient $p$, on transport properties of a CNT-based spin valve with two identical tunnel barriers. (a) Spindependent transmission coefficient $\mathcal{T}_{\sigma}$ and (b) interfacial phase shift $\varphi_{\sigma}$ of a single barrier shown as functions of the barrier strength $Z$. Here, $\sigma=\uparrow(\downarrow)$ refers to spin-majority (-minority) electrons. For a description of the lines, see the legend in (a). (c) Dependence of the tunneling magnetoresistance (TMR) on the barrier strength $Z$ for $E_{g} / \Delta \varepsilon_{r} \approx 0.09$ - the solid line is identical to the solid line in Fig. 2(b). (d) TMR plotted as a function of $E_{g} / \Delta \varepsilon_{r}$ for $Z=1$ - the solid line is identical to the long-dashed line in Fig. 2(c). The corresponding lines in (c) and (d) represent the same value of $p$ as given in (c). The remaining parameters are the same as in Fig. 2.

mind that the length and the barrier strength will affect the tuning of the TMR effect with the gate voltage. In general, it can be seen that the maxima in conductance, and consequently also in a TMR signal, arise owing to the phase factor $\theta_{\sigma}^{c}(\varepsilon)$ occurring in the transmission coefficient (19). It is, thus, essential to keep track of this phase when simulating experimental data, and the present approach, which straightforwardly relates both the interface transmission (16) and the interfacial phase shift (20) to the strength of a tunnel barrier forming at the interface, proves to be useful for doing it consistently.

To understand how the strength of the tunnel barriers affects the TMR, as shown in Figs. 2(a) and 2(b), let us analyze the dependence of the spin-dependent transmission $\mathcal{T}_{\sigma}$ and the interfacial phase shift $\varphi_{\sigma}$ of a single tunnel barrier; see Figs. 3(a) and 3(b). First, in Fig. 2(a), one can distinguish three generic regions with respect to the barrier strength $Z$ : for small $Z \lesssim 0.1$ and large $Z \gtrsim 10$ regions where the TMR remains roughly constant, and a transitional region $(0.1 \lesssim Z \lesssim 10)$ where TMR changes significantly. Interestingly, the occurrence of these regions can be 
explained by considering the behavior of $\mathcal{T}_{\sigma}$ and $\varphi_{\sigma}$ as a function of $Z$.

For a small $Z$, a single barrier is characterized by a high transmission coefficient, with $\mathcal{T}_{\uparrow} \neq \mathcal{T}_{\downarrow}$ if $p \neq 0$ [Fig. 3(a)], and the interfacial phase shifts being close to $-\pi$ [Fig. 3(b)]. As a result, resonances in the TMR, which originate from $\mathcal{T}_{\uparrow} \neq \mathcal{T}_{\downarrow}$, become only weakly shifted with respect to $E_{g}$ as $Z$ is increased; see Eq. (22). Note that, even in the absence of spin polarization $(p=0)$, the interface does not become fully transparent; that is, the transmission coefficient is still less than 1 [see the solid line in Fig. 3(a)]. This result has its origin in the mismatch of the electronic band structure between a lead and a CNT, which effectively manifests as different wave vectors for the lead, $k_{c\left(\sigma_{q}\right)}$, and the CNT, $k_{n}^{w}$, in Eq. (16). A further increase of $Z$ into the transitional region leads to a rapid drop of $\mathcal{T}_{\sigma}$, and to an increase of $\varphi_{\sigma}$. The maximum of $\varphi_{\sigma}$ shifts with $Z$ depending on $p$ and $\sigma$ [Fig. 3(b)]. Notably, in this region a significant difference between $\varphi_{\uparrow}$ and $\varphi_{\downarrow}$ develops, which, in turn, means that resonant energies $\tilde{\varepsilon}_{\sigma p}^{c}$ get markedly different for different spin orientations and magnetic configurations. This difference, in combination with the fact that, in the $Z$ range under consideration, a transition from $\mathcal{T}_{\uparrow}<\mathcal{T}_{\downarrow}$ to $\mathcal{T}_{\uparrow}>\mathcal{T}_{\downarrow}$ occurs, leads to great changes in the TMR preceded with a large shift of the resonances with respect to $E_{g}$. Finally, for large $Z$ 's, the barriers become almost nontransparent, with the interfacial phase shift again approaching $-\pi$ and $\varphi_{\uparrow}=\varphi_{\downarrow}$. Consequently, for asymptotically large Z's, one observes a constant TMR with narrow resonant dips appearing at exactly the same values of $E_{g}$ as the resonant peaks in the limit of $Z \rightarrow 0$.

To conclude the present discussion, in Figs. 3(c) and 3(d), we additionally show how the main features of the TMR as a function of $Z$ considered above depend on the spin polarization of the electrodes. The TMR effect increases with the polarization of the contacts for strong barriers just as in conventional spin valves; see Fig. 3(c). Interestingly, the nontrivial behavior of the TMR around $Z=1$ is also more pronounced for a larger polarization and, thus, the tunability of the TMR with the gate voltage as shown in Fig. 3(d).

Finally, we would like to comment on the behavior of the TMR in the limit of $Z \rightarrow 0$. In general, one expects that, in the experimental situation of electrical spin (diffusive) injection from a ferromagnet into a nonmagnetic material, the spin polarization of the injected current can be quenched due to the conductance mismatch of these two materials - the effect being especially pronounced if the spin injection occurs in a semiconductor (SC) [53,54]. Moreover, the conductance mismatch then essentially means that the transmission coefficient becomes spin independent. This problem, however, can be circumvented if a spin-dependent interface resistance (e.g., due to a tunnel barrier), with some threshold value related to the resistivity and spin-diffusion length of a SC, is introduced [55,56]. In the present considerations, on the other hand, such an effect is not captured by the model under investigation, that is, a CNT treated as a ballistic 1D conductor. Here, it is assumed that, once an electron is tunneled into the CNT, its spin remains coherent until it tunnels out, which basically corresponds to the situation of both the spin-diffusion length and the mean free path being sufficiently long. As shown by Valet and Fert [57], in such a ballistic limit, the usage of the Landauer approach is justified, without the need of applying the description of spin-dependent electrochemical potentials by means of the diffusion equation. Importantly, for that reason, in the current case, the spin dependence is preserved also in the limit of a vanishingly small tunnel barrier and, consequently, a nonzero TMR signal is obtained. We note that a similar effect was also derived for a spin injection into a SC in a ballistic picture [30,31].

\section{Limit of strong tunnel barriers}

In order to develop the complete physical picture, let us now briefly analyze transport in the case of a large $Z$, which has already been widely studied $[25,28,29]$. To begin with, in such a limit, one generally derives

$$
\mathcal{T}_{\sigma}^{q c} \approx \mathbb{T}_{\sigma}^{q} \sqrt{1+2 c\left(\sigma_{q}\right) p}
$$

with

$$
\mathbb{T}_{\sigma}^{q}=\frac{\mathbb{T}_{q}}{\left(1+\eta_{\sigma} \alpha_{q}\right)^{2}} \quad \text { and } \quad \mathbb{T}_{q}=\frac{4}{\left(Z_{q}\right)^{2}} \frac{v_{F}^{w}}{v_{0}},
$$

where $v_{0}=\sqrt{2 E_{F} / m_{e}}$. In the equations above, $\mathbb{T}_{\sigma}^{q}$ represents the transmission coefficient of the $q$ th interface $(q=L, R)$ whose spin dependence stems exclusively from the spin selectiveness of the barrier. This effect is analyzed in full detail in Sec. VA 3, and in the following discussion we assume spin nonselective barriers $\left(\alpha_{q}=0\right)$. Interestingly, in such a case and for a small degree of spin polarization of electrodes, one obtains $\mathcal{T}_{\sigma}^{q} \approx \mathbb{T}^{q}\left(1+\eta_{\sigma} p\right)$. Moreover, in the limit of weakly transparent barriers, $\mathcal{T}_{\sigma}^{q c} \ll 1$, and expanding $\cos \left[\theta_{\sigma}^{c}(\varepsilon)\right]$ around the resonant energy $\tilde{\varepsilon}_{\sigma p}^{c}$, one finds that the expression for the transmission coefficient $\mathscr{T}_{\sigma}^{c}(\varepsilon)$ of the device takes the form of the Breit-Wigner formula [58,59]

$$
\mathscr{T}_{\sigma}^{c}(\varepsilon)=\mathscr{T}_{\max , \sigma}^{c} \frac{\left(\Gamma_{\sigma}^{c}\right)^{2} / 4}{\left(\varepsilon-\tilde{\varepsilon}_{\sigma p}^{c}\right)^{2}+\left(\Gamma_{\sigma}^{c}\right)^{2} / 4} .
$$

In the equation above, $\Gamma_{\sigma}^{c}=\Gamma_{\sigma}^{L c}+\Gamma_{\sigma}^{R c}$, and $\mathscr{T}_{\max , \sigma}^{c}=$ $4 \Gamma_{\sigma}^{L c} \Gamma_{\sigma}^{R c} /\left(\Gamma_{\sigma}^{c}\right)^{2}$ denotes the maximal value of the transmission coefficient at resonance, whereas $\Gamma_{\sigma}^{q c}=\hbar \nu \mathcal{T}_{\sigma}^{q c}$ is the decay width of the resonant level due to the tunneling of electrons with spin $\sigma$ through the $q$ th interface. It is expressed in terms of the attempt frequency $\nu$ defined as 
$\nu^{-1}=\left.\hbar\left[d \theta_{\sigma}^{c}(\varepsilon) / d \varepsilon\right]\right|_{\varepsilon=\tilde{\varepsilon}_{\sigma p}^{c}}=2 \ell / v_{F}^{w}[60]$, which basically describes the number of chances per unit time an electron that enters a CNT through the $q$ th interface has to leave it through the same interface.

Using Eq. (25) with Eq. (18), one can then find the asymptotic values of the TMR for large $Z$ 's to be (i) off resonance, i.e., when $\varepsilon-\tilde{\varepsilon}_{\sigma p}^{c} \gg \Gamma_{\sigma}^{c} / 2$,

$$
\mathrm{TMR}_{\text {off-res }}=\frac{1-\sqrt{1-4 p^{2}}}{\sqrt{1-4 p^{2}}},
$$

which for $p=0.25$ yields $\mathrm{TMR}_{\text {off-res }} \approx 15.5 \%$; (ii) at resonance, i.e., when $\varepsilon=\tilde{\varepsilon}_{\sigma p}^{c}$,

$$
\mathrm{TMR}_{\text {res }}=\frac{1}{2} \mathrm{TMR}_{\text {off-res }} .
$$

The variation of TMR between these two limiting values can be seen as a double-dotted-dashed line in Figs. 2(c), where the dips correspond to resonant tunneling of electrons-this variation also manifests as peaks in conductance given by the double-dotted-dashed line in Fig. 2(f).

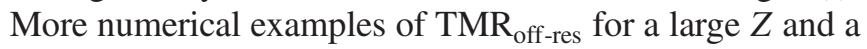
different $p$ can be seen in Fig. 3(c). Furthermore, it is worth noting that if, in derivation of the formula above instead of Eq. (23), one employs its counterpart for low spin polarizations of electrodes, the Jullière value of tunneling magnetoresistance [61], $\mathrm{TMR}_{\text {off-res }}=2 p^{2} /\left(1-p^{2}\right)$, is recovered.

Another observation one can make is that the position of the resonances in conductance in Fig. 2(d) is independent of $Z$ for large $Z$ 's, whereas as $Z$ gets diminished, their position becomes sensitive to $Z$. As already mentioned, this effect stems from the fact that, when $Z$ increases, the spindependent interfacial shifts $\varphi_{\sigma}^{q}$ for both spin orientations become equal at some point, and for even larger Z's they remain independent of the barrier strength, taking a constant value of $-\pi$, as can be seen in Fig. 3(b). Furthermore, it is clear that, for almost fully transparent (very-small-Z) and nontransparent (large- $Z$ ) interfaces, the part of the phase factor $\theta_{\sigma}^{c}(\varepsilon)$ in Eq. (19) corresponding to the spindependent interfacial phase shift is $\varphi_{\sigma}^{L c}+\varphi_{\sigma}^{R c} \approx-2 \pi$ [see Fig. 3(b)], regardless of the magnetic configuration of the spin valve. In contrast, for the intermediate regime of the barrier strength $Z$, where $\varphi_{\sigma}^{L c}+\varphi_{\sigma}^{R c}<-2 \pi$, this phase shift is different for the parallel $(c=\mathrm{P})$ and antiparallel ( $c=\mathrm{AP}$ ) magnetic configurations, so that the effect of spin-dependent backscattering of electrons into a CNT becomes visible in the TMR signal. For this reason, it is justified to neglect the spin-dependent interfacial phase shift for very small and large Z's, and one can use this phase shift as an indication for an intermediate barrier strength $(0.1 \lesssim Z \lesssim 10)$.

To complete the discussion of asymptotic values of the TMR for a large $Z$, we note that one should be careful when estimating the spin-polarization coefficient $p$ of the electrodes. If one adjusts the gate voltage in such a way that the device is in the transport regime close to the resonant one but still off resonant [cf. the dashed lines in Fig. 4(a)], the TMR signal can become dependent on temperature; see Fig. 4. Specifically, the thermal broadening of the resonant peak in conductance leads also to a wider dip in the TMR, as shown in Fig. 4(a). When analyzing the TMR as a function of the barrier strength $Z$ [Fig. 4(b)], this broadening, in turn, can be observed for large $Z$ 's as a thermally induced transition of the TMR between the two limiting values discussed above. Since the period of the oscillations $\Delta \varepsilon_{r}$ is inversely proportional to the length $\ell$ of a CNT, one expects such an effect of temperature on the TMR to be more profound for longer CNTs.

\section{Asymmetry of tunnel barriers}

Let us now go beyond the assumption that both of the tunnel barriers are identical and consider the asymmetrical situation $\left(Z_{L} \neq Z_{R}\right)$. This situation is illustrated in Fig. 5(a), which in a similar fashion to Fig. 2(a) presents the evolution of the TMR in response to increasing now only the strength of the right barrier $Z_{R}$, while the strength of the left barrier is kept constant, $Z_{L}=1$. Note that, for the sake of clarity, only one period in $E_{g} / \Delta \varepsilon_{r}$ has been plotted here. Noticeably, while, for a vanishingly small right barrier $\left(Z_{R} \ll Z_{L}\right)$, the TMR remains qualitatively the same as in the case of the symmetric barriers, for the strong asymmetry of tunnel barriers - that is, $Z_{R} \gg Z_{L}$-a significant modification of the TMR is observed. Specifically, a distinctive sawlike pattern develops in this limit with large negative values of the TMR; see Fig. 5(c). In fact, such an asymmetry in the strength of the tunnel barriers was essential to take into account in order to explain the
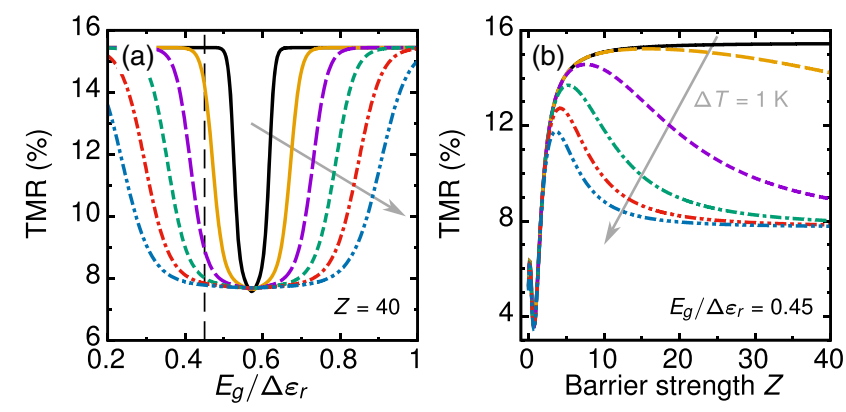

FIG. 4. (a) Dependence of the tunneling magnetoresistance (TMR) on temperature in the vicinity of the resonant transmission in the case of a large barrier strength $(Z=40)$. The solid line corresponds to $T=1 \mathrm{~K}$ and the gray arrow indicates the increase of temperature with the step of $\Delta T=1 \mathrm{~K}$ up to $6 \mathrm{~K}$ for the double-dotted-dashed line. (b) Evolution of the TMR as a function of the barrier strength $Z$ shown for different tempeatures and $E_{g} / \Delta \varepsilon_{r}=0.45$, which is schematically represented by a vertical dashed line in (a). Note that the same pattern scheme for temperatures as in (a) is used. 

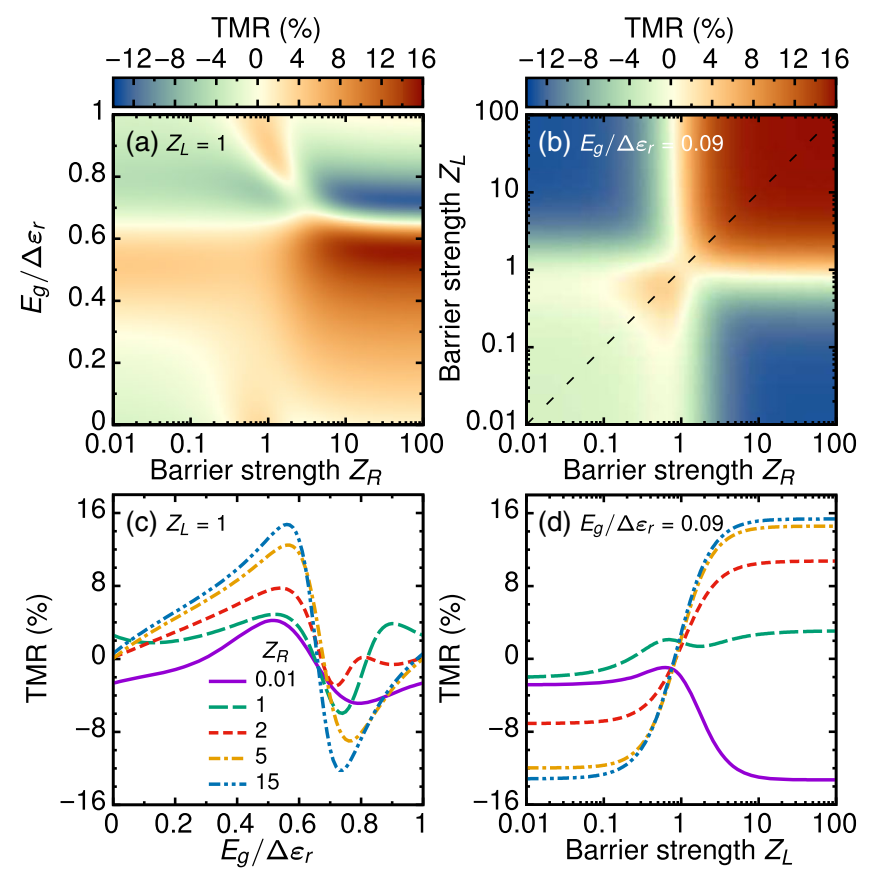

FIG. 5. The effect of the left-right barrier-strength asymmetry on the tunneling magnetoresistance (TMR). (a) Analogous to Fig. 2(a) except that, at present, the strength of only the right barrier $Z_{R}$ is changed over one period $\Delta \varepsilon_{r}$ for $Z_{L}=1$. (b) Density map of $\operatorname{TMR}\left(Z_{R}, Z_{L}\right)$ for $E_{g} / \Delta \varepsilon_{r} \approx 0.09$, which corresponds to the off-resonance limit for a large $Z$, and, in particular, to the position in the middle between two neighboring dips in the TMR [see the double-dotted-dashed line in Fig. 2(c)]. Note that the bright color in (a) and (b) represents $\mathrm{TMR} \approx 0$, whereas the dashed line in (b) denotes the symmetric case of $Z_{L}=Z_{R}$. (c) Cross sections of (a) for indicated values of the right barrier strength $Z_{R}$. Here, the long-dashed (green) line represents the symmetric case $\left(Z_{L}=Z_{R}\right)$; cf. Fig. 2(c). (d) Dependence of the TMR on $Z_{L}$ shown for $E_{g} / \Delta \varepsilon_{r} \approx 0.09$ and values of $Z_{R}$ given in the legend of (c)-i.e., the vertical cross sections of (b). All other parameters are the same as in Fig. 2.

occurrence of a negative TMR signal in the experimental study of a spin-polarized transport through a CNT by Sahoo et al. [26] — see the lines for $Z_{R}=5$ (dotted dashed) and $Z_{R}=15$ (double dotted dashed) in Fig. 5(c), which qualitatively reproduces their result.

Next, to gain a better insight into how the asymmetry of the barriers affects the TMR, in Fig. 5(b) we show the dependence of the TMR on the strength of both the left $\left(Z_{L}\right)$ and right $\left(Z_{R}\right)$ barriers for the gate-induced energy shift $E_{g} / \Delta \varepsilon_{r}=0.09$ corresponding to the off-resonant limit from Fig. 2(a). The dashed line serves here merely as a guide for the eye denoting the case of identical barriers, with corresponding cross sections along this line given by a solid curve in Fig. 2(b). Departing in either direction perpendicular to the dashed line represents the situation where one of the barriers increases, whereas the other one gets smaller and smaller. A dramatic change in the TMR occurs when one of the barriers becomes very small.
Noticeably, the TMR can take then large negative values, which means that the device displays higher conductance in the antiparallel magnetic configuration of the electrodes.

Employing the Breit-Wigner formula (25) for the situation when the strength of one tunnel barrier is significantly larger than the other one (i.e, asymmetric barriers, referred to as "as") and assuming, e.g., $Z_{R} \gg Z_{L}$, which corresponds to $\Gamma_{\sigma}^{L c} \gg \Gamma_{\sigma}^{R c}$ [recall that $\Gamma_{\sigma}^{q c} \propto \mathcal{T}_{\sigma}^{q c}$ and $\mathcal{T}_{\sigma}^{q c} \propto 1 /\left(Z_{q}\right)^{2}$; see Eqs. (23) and (24)], we find the asymptotic form for the TMR at resonance,

$$
\mathrm{TMR}_{\mathrm{res}}^{\mathrm{as}}=\sqrt{1-4 p^{2}}-1,
$$

whereas the low-spin-polarization expression for the transmission coefficients of the barriers yields $\mathrm{TMR}_{\mathrm{res}}^{\mathrm{as}}=-2 p^{2} /$ $\left(1+p^{2}\right)$, in agreement with previous studies [29]. On the other hand, in the off-resonant case, the analogous asymptotic formula for $\mathrm{TMR}_{\text {off-res }}^{\text {as }}$ is identical to Eq. (26). Importantly, we recall that these two asymptotic expressions for the TMR are, in general, valid only if $Z_{L}$, $Z_{R} \gtrsim 10$, that is, for weakly transparent barriers $\left(\mathcal{T}_{\sigma}^{q c} \ll 1\right)$; cf. Fig. 3(a). Nevertheless, one can already see that the negative value of the TMR in Fig. 5(a) is very close to $\mathrm{TMR}_{\text {res }}^{\mathrm{as}}$, whereas, in Fig. 5(b), the asymptotic value $\mathrm{TMR}_{\text {off-res }}^{\text {as }}$ is reached as soon as $Z_{L}, Z_{R}>2$ (see the topright corner of the plot). As one can see in Fig. 5(c), the tunability with respect to the gate response of the TMR signal is strongest in the asymmetric case, if one barrier is very strong (here $Z_{R}=15$ ), while the strength of the second barrier assumes a value of about $Z_{L}=1$.

Concluding the results for barriers without spin selectivity, it is now clear that the largest TMR signal of $15.5 \%$ is obtained if a device with realistic parameters, as specified at the beginning of Sec. V, is tuned to be off resonant and if the tunnel barriers are strong $(Z \gtrsim 10)$. Additionally, the response to a gate voltage is strongest if the barriers are asymmetric (again tuned off resonant). For instance, for $Z_{L}=1$ and $Z_{R}=15$ [see the double-dotted line in Fig. 5(c)] and assuming a realistic gate coupling of $c_{\text {gate }}=0.33$, the TMR signal can be tuned from approximately $14 \%$ to approximately $-12 \%$ within a $5-\mathrm{mV}$ shift of the gate voltage.

Such devices can be fabricated using CoPd as ferromagnetic leads that mainly show low or intermediate tunnel barriers with $Z \lesssim 10$ (cf. Ref. [10]), adding a thin insulating layer between the CNT and one contact (both contacts) for asymmetric (symmetric) barriers. If a spin-selective insulator is used, the barriers will, additionally, become spin selective.

\section{Spin-selective barriers}

Finally, we address the situation where tunnel barriers at the interfaces between electrodes and a CNT are, additionally, spin selective, that is, $\alpha_{L} \neq 0$ and/or $\alpha_{R} \neq 0$. Such a situation can arise when spin-selective insulators like $\mathrm{EuO}$ 
[62] or EuS [63] or chiral molecules [64-66] are used as tunnel barriers. For the simplicity of the following discussion, we return to the situation of the symmetric barriers $\left(Z_{L}=Z_{R}=Z\right)$, and only at the end of the section do we consider the case of asymmetric barriers $\left(Z_{L} \neq Z_{R}\right)$, which is expected to be more common for real devices.

Numerical results illustrating how the spin selectiveness of tunnel barriers affects the TMR are shown in Fig. 6 for identical barriers $\left(\alpha_{L}=\alpha_{R}=\alpha\right)$. Adding insulators between the CNT and the ferromagnetic leads will increase the barrier strength. Therefore, in our discussion, we will focus on large tunnel barriers $(Z \gtrsim 10)$. As is visible in Figs. 6(a) and 6(d) [cf. Fig. 2(a)], tunnel barriers that filter
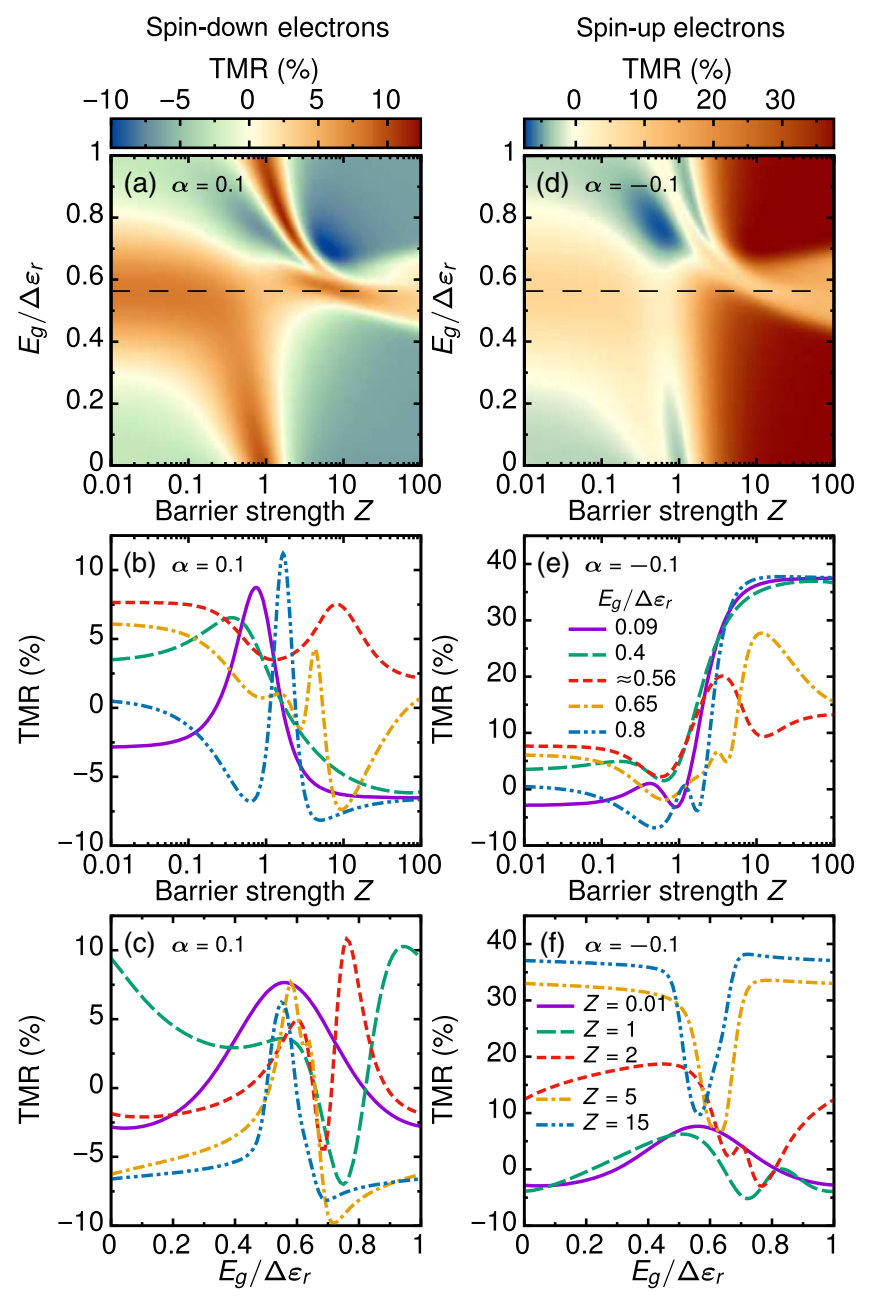

FIG. 6. Analogous to Figs. 2(a)-2(c), except that it is assumed here that the tunnel barriers are spin selective $\left(\alpha_{L}=\alpha_{R}=\alpha\right)$. Results for two different values of the barrier-spin-asymmetry parameter $\alpha$ are shown: for $\alpha=0.1$ in panels (a)-(c), where spindown electrons are preferred, and for $\alpha=-0.1$ in panels (d)-(f), where spin-up electrons are preferred. Note that, to facilitate the comparison of (a) to (d), the color scale in (a) matches that of (d) in the corresponding range of the TMR. Finally, we assume here again that $Z_{L}=Z_{R}=Z$ and that the remaining parameters are the same as in Fig. 2. incident electrons based on their spin orientation lead to significant, both qualitative and quantitative, changes in the TMR signal which become especially visible at large barrier strength $Z$. Furthermore, this spin-filtering process, characterized by the spin-asymmetry parameter $\alpha$ [Eq. (17)], depends essentially on whether more spin-up $[\alpha<0$, as in Figs. 6(d), 6(e), and 6(f)] or spin-down [ $\alpha>0$, as in Figs. 6(a), 6(b), and 6(c)] electrons are passed through the barriers. Note that the spin orientation is defined with respect to the majority spins of the left electrode, which are defined as spin up (cf. Fig. 1). Since the main quantitative difference between the two cases under discussion occurs in the limit of transitional and large Z's [for small Z's, there are neither qualitative nor quantitative differences between Figs. 6(a) and 6(d) - note the different scale ranges for the TMR], it may be instructive at this point to derive some asymptotic expressions for the TMR.

We use the Breit-Wigner formula, Eq. (25), to derive the following asymptotic expressions. In the off-resonance limit for two symmetric barriers (referred to by a superscript "s"), i.e., $\alpha_{L}=\alpha_{R}=\alpha$, one obtains

$$
\mathrm{TMR}_{\text {off-res }}^{\mathrm{s}}=\mathrm{TMR}_{\text {off-res }}+\Delta \mathrm{TMR}_{\text {off-res }}^{\mathrm{s}},
$$

with

$$
\Delta \mathrm{TMR}_{\text {off-res }}^{\mathrm{s}}=-\frac{4 p}{\sqrt{1-4 p^{2}}} \mathcal{F}_{1}(\alpha)
$$

and

$$
\mathcal{F}_{1}(\alpha)=\frac{2 \alpha\left(1+\alpha^{2}\right)}{4 \alpha^{2}+\left(1+\alpha^{2}\right)^{2}} .
$$

On the other hand, at resonance for identical barriers, one finds that

$$
\mathrm{TMR}_{\mathrm{res}}^{\mathrm{s}}=\mathrm{TMR}_{\text {res }}
$$

which basically means that the resonant transport of electrons through the device is insensitive to the spin selectiveness of the tunneling barriers, a fact that is discussed in more detail at the end of this section.

In general, if at least one barrier is spin selective, this characteristic leads to a correction to the off-resonance TMR. This correction is determined both by the spin polarization of the electrodes $p$ and by the spin asymmetry of the barriers $\alpha$. What is more, the correction is positive [negative] if spin-up $(\alpha<0)$ [spin-down $(\alpha>0)$ ] electrons are preferred. In the following, we assume the spin selectivity of the barriers to be $|\alpha| \leq 0.25$, which is a very moderate choice regarding the fact that, for $\mathrm{EuO}$, a spinfilter efficiency as large as $80 \%$ has been observed in tunnel junctions [62]. It can be checked that, for $p=0.25$, one expects to achieve a TMR signal up to $\mathrm{TMR}_{\text {off-res }}^{\mathrm{s}} \approx 37 \%$ for strong barriers and spin-up electrons [see Fig. 6(e)] and corrections as large as $\Delta \mathrm{TMR}_{\text {off-res }}^{\mathrm{s}} \approx 40 \%$ compared to 
$\mathrm{TMR}_{\text {off-res }}$. Also, the gate-voltage response of the TMR signal is strongest for strong barriers [see Fig. 6(f)], and tuning between $10 \%$ and $37 \%$ within a gate voltage of $5 \mathrm{mV}$, assuming again a gate coupling $c_{\text {gate }}=0.33$. In contrast to spin-up electrons, the maximum value, as well as the strongest gate response for the TMR signal for a spinselective barrier that prefers spin-down electrons, is, in total, not only smaller but also found for small or intermediate barrier strength [see Figs. 6(b) and 6(c)]. Importantly, note that the spin moment of EuS aligns antiferromagnetically with respect to the spin moment of $\mathrm{Co}$ in Co/EuS multilayers [67]. For this reason, using EuS as a spin-selective barrier with ferromagnetic leads from CoPd will, most likely, lead to a selection of spin-down electrons.

Though the fabrication of such a device is more tedious compared to symmetric barriers, it is possible to have only one spin-selective barrier $q$, i.e., $\alpha_{L}=\alpha$ and $\alpha_{R}=0$ for $q=L$ or $\alpha_{L}=0$ and $\alpha_{R}=\alpha$ for $q=R$, and in the offresonant case, one obtains

$$
\mathrm{TMR}_{\text {off-res }}^{q}=\mathrm{TMR}_{\text {off-res }}+\Delta \mathrm{TMR}_{\text {off-res }}^{q},
$$

with

$$
\Delta \mathrm{TMR}_{\text {off-res }}^{q}=-\frac{4 p}{\sqrt{1-4 p^{2}}} \mathcal{F}_{2}(\alpha)
$$

and

$$
\mathcal{F}_{2}(\alpha)=\frac{\alpha}{1+\alpha^{2}}
$$

Clearly, only the dependence on $\alpha$ is affected by whether one or both barriers are spin selective [cf. Eqs. (30) and (34)]. For $\alpha \neq 0$ and $|\alpha|<1$, one gets $\left|\mathcal{F}_{1}(\alpha)\right|>\left|\mathcal{F}_{2}(\alpha)\right|$, and the change in the TMR signal is reduced to $\Delta \mathrm{TMR}_{\text {off-res }}^{L / R} \approx 25 \%$.

However, if only one barrier is spin selective, the resonant TMR signal is also changed:

$$
\mathrm{TMR}_{\mathrm{res}}^{q}=\mathrm{TMR}_{\mathrm{res}}+\Delta \mathrm{TMR}_{\mathrm{res}}^{q},
$$

where

$\Delta \mathrm{TMR}_{\mathrm{res}}^{q}=\frac{1}{2}\left[\mathcal{F}_{3}(\alpha)-1\right]+\frac{\mathcal{F}_{3}(\alpha) \mathcal{F}_{4}^{q}(\alpha, p)-1}{2 \sqrt{1-4 p^{2}}}$,

with

$$
\mathcal{F}_{3}(\alpha)=\frac{16+4 \alpha^{2}\left[\alpha^{2}\left(1+\alpha^{2}\right)-4\right]}{\left(4+\alpha^{4}\right)^{2}}
$$

and

$$
\mathcal{F}_{4}^{L / R}(\alpha, p)=\frac{2 \sqrt{1-4 p^{2}}\left(1+\alpha^{2} \mp 4 p \alpha\right)\left[1+\left(1-\alpha^{2}\right)^{2}\right]+\left(1-4 p^{2}\right)\left[1+\left(1-\alpha^{2}\right)^{4}\right]+\sum_{\eta= \pm}(1+\eta 2 p)^{2}(1 \mp \eta \alpha)^{4}}{2\left(1+\alpha^{2} \mp 4 p \alpha\right)\left[1+\left(1-\alpha^{2}\right)^{2}\right]+4 \sqrt{1-4 p^{2}}\left(1-\alpha^{2}\right)^{2}}
$$

Note that $\lim _{\alpha \rightarrow 0} \mathcal{F}_{3}(\alpha)=1$ and $\lim _{\alpha \rightarrow 0} \mathcal{F}_{4}^{L / R}(\alpha, P)=1$, so that, in the limit of vanishingly small spin selectiveness of barriers, we recover the previously found result: that is, $\mathrm{TMR}_{\mathrm{res}}^{q}=\mathrm{TMR}_{\text {res }}$.

Figure 7 presents the evolution of the off-resonance TMR as a function of the barrier strength $Z$ for selected values of the spin-asymmetry parameter $\alpha$ in three specific cases: (a) and (b) when both tunnel barriers are identical $\left(\alpha_{L}=\alpha_{R}\right)$ or when only one of the barriers is spin selective: the left in (c) and (d) and the right in (e)-(f). There is no dependence of the TMR on $\alpha$ seen for a small $Z$, whereas, for a large $Z$, a significant variation of the TMR occurs, with the asymptotic values of the TMR given by the expressions above. Moreover, in the latter limit one observes a general trend that, for positive $\alpha$ (spin-down electrons preferred), the TMR decreases, so that for a sufficiently large $\alpha$, it can get negative, whereas for a negative $\alpha$ (spin-up electrons preferred), the TMR increases. Interestingly, for the transitional values of $Z$, we find that the TMR varies nonmonotonically in the case with identical barriers and only the right barrier being spin selective. On the other hand, in the case with only the left barrier being spin selective, the TMR remains rather unaffected by $\alpha \neq 0$, and only as $Z$ is further increased does the TMR start to gradually approach its asymptotic values. As shown previously, this behavior can be understood in terms of a spin-dependent transmission coefficient and interfacial phase shift for a single tunnel barrier. Importantly, if only the left barrier is spin selective, its effect is the same for both magnetic configurations of electrodes, so that the TMR is only slightly influenced. This result is due to the fact that the orientation of the spin moment of the left electrode defines here the reference frame. The situation is different when the right barrier is spin selective. In such a case, depending on the magnetic configuration, the barrier prefers either spin-up or spin-down electrons, and thus conductances in both magnetic configurations are affected differently, which ultimately reveals itself in the TMR signal.

Finally, we note that, in real devices, one should generally expect that the combination of the two effects studied above will occur; that is, the two tunnel barriers will be asymmetric in terms of both strength $\left(Z_{L} \neq Z_{R}\right)$ and spin selectiveness $\left(\alpha_{L} \neq \alpha_{R}\right)$. We find that, in such a case, the previously derived asymptotic formulas 

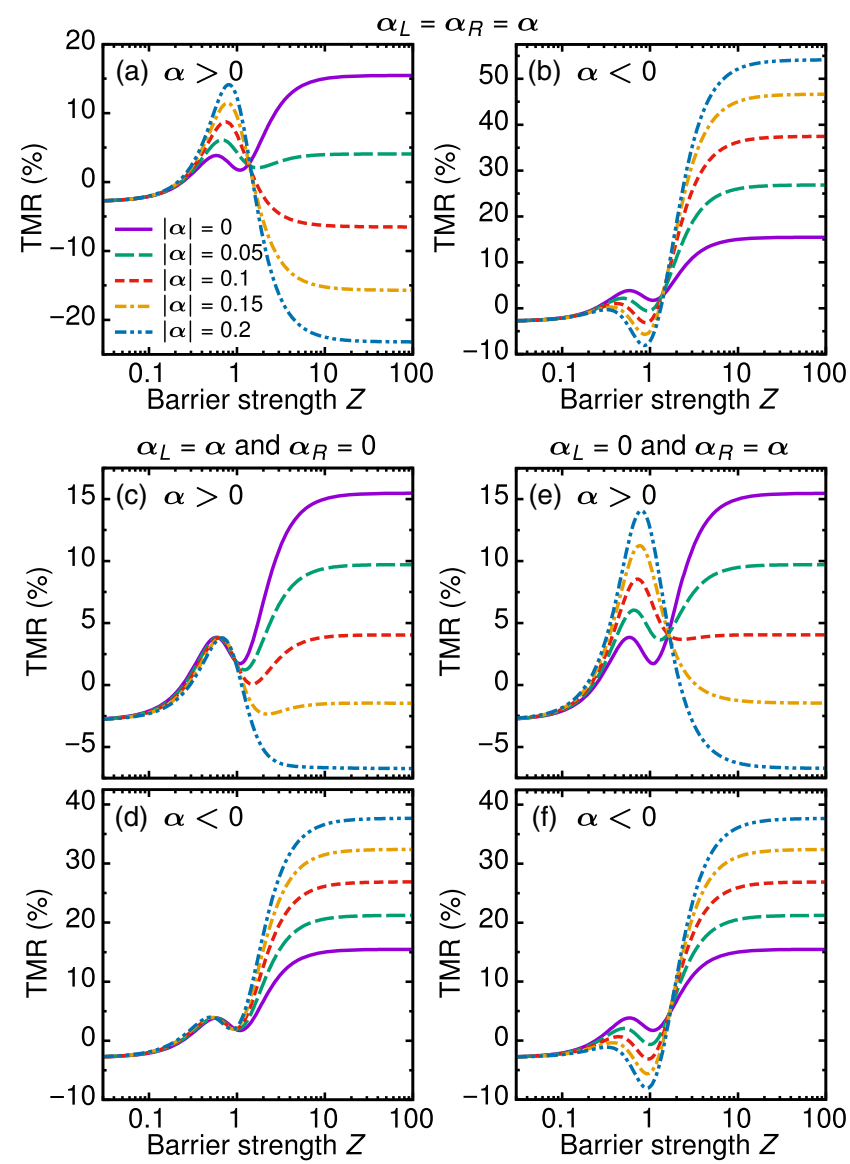

FIG. 7. The effect of the spin selectivity of the tunnel barriers on the tunneling magnetoresistance (TMR) shown as a function of the barrier strength $Z$ for $E_{g} / \Delta \varepsilon_{r}=0.09$ and several values of the spin-asymmetry parameter $\alpha$. (a),(b) Represent the situation with identical barriers $\left(\alpha_{L}=\alpha_{R}=\alpha\right)$, whereas, in (c)-(f), the case of only one barrier being spin selective is shown - that is, the left one $\left(\alpha_{L}=\alpha\right.$ and $\left.\alpha_{R}=0\right)$ in (c) and (d) and the right one $\left(\alpha_{L}=0\right.$ and $\left.\alpha_{R}=\alpha\right)$ in (e)-(f). Note that the solid line corresponds to $\alpha=0$ and serves as the reference line for the comparison of different plots. This line is also identical to the solid line in Fig. 2(b). All other parameters as the same as in Fig. 2.

for strongly asymmetric barriers $\left(Z_{R} \gg Z_{L}\right)$ - see Sec. VA 2 -become modified as follows to incorporate the effect of different spin-selective properties of each barrier (we use a prime to distinguish this case): off resonance, one obtains

$$
\left(\mathrm{TMR}_{\mathrm{off-res}}^{\mathrm{as}}\right)^{\prime}=\mathrm{TMR}_{\mathrm{off-res}}+\Delta \mathrm{TMR}_{\mathrm{off}-\text { res }}^{\mathrm{as}},
$$

with

$$
\Delta \mathrm{TMR}_{\text {off-res }}^{\text {as }}=-\frac{4 p}{\sqrt{1-4 p^{2}}} \mathcal{S}_{+}\left(\alpha_{L}, \alpha_{R}\right)
$$

whereas, at resonance, one gets

$$
\left(\mathrm{TMR}_{\mathrm{res}}^{\mathrm{as}}\right)^{\prime}=\frac{\mathrm{TMR}_{\mathrm{res}}^{\mathrm{as}}}{1-4 p \mathcal{S}_{-}\left(\alpha_{L}, \alpha_{R}\right)}+\Delta \mathrm{TMR}_{\mathrm{res}}^{\mathrm{as}}
$$

with

$$
\Delta \mathrm{TMR}_{\mathrm{res}}^{\mathrm{as}}=\frac{4 p \mathcal{S}_{-}\left(\alpha_{L}, \alpha_{R}\right)}{1-4 p \mathcal{S}_{-}\left(\alpha_{L}, \alpha_{R}\right)} .
$$

The coefficient $\mathcal{S}_{ \pm}\left(\alpha_{L}, \alpha_{R}\right)$, defined as

$$
\mathcal{S}_{ \pm}\left(\alpha_{L}, \alpha_{R}\right)=\frac{\left(\alpha_{L} \pm \alpha_{R}\right)\left(1 \pm \alpha_{L} \alpha_{R}\right)}{\left(\alpha_{L} \pm \alpha_{R}\right)^{2}+\left(1 \pm \alpha_{L} \alpha_{R}\right)^{2}}
$$

describes the asymmetry of the tunnel barriers due to the difference in spin-asymmetry parameters between the left $\left(\alpha_{L}\right)$ and the right $\left(\alpha_{R}\right)$ barrier. One can then notice that, for the symmetric case-that is, when $\alpha_{L}=\alpha_{R}=\alpha$-one obtains $\mathcal{S}_{+}(\alpha, \alpha) \equiv \mathcal{F}_{1}(\alpha)$ [see Eq. (31)], so that the asymptotic equations for the TMR given by Eqs. (29) and (40) become identical. Similarly, one finds the relation between Eqs. (33) and (40) for only a single barrier being spin selective, $\mathcal{S}_{+}(\alpha, 0)=\mathcal{S}_{+}(0, \alpha) \equiv \mathcal{F}_{2}(\alpha)$; see Eq. (35). The analysis of $\mathcal{S}_{+}\left(\alpha_{L}, \alpha_{R}\right)$ brings us to the conclusion that $\left(\mathrm{TMR}_{\text {off-res }}^{\text {as }}\right)^{\prime}$ can be effectively maximized by ensuring that the barriers are symmetric $\left(\alpha_{L}=\alpha_{R}\right)$ and engineering them in such a way that spin-up electrons are favored (i.e., $\left.\alpha_{L}, \alpha_{R}<0\right)$.

On the other hand, in the resonant case, we notice that if both barriers are identical $\left(\alpha_{L}=\alpha_{R}=\alpha\right)$, the spin selectiveness of the barriers plays no role, as $\mathcal{S}_{-}(\alpha, \alpha)=0$ and Eq. (28) is recovered. This striking difference can be qualitatively understood by considering how the spin selectiveness of the barriers affects the conductance. In the case of the strongly asymmetric barriers under discussion, one finds that the spin-resolved conductance in the magnetic configuration $c=\mathrm{P}$, AP depends on the transmission coefficients (23) of the left $\left(\mathcal{T}_{\sigma}^{L c}\right)$ and right $\left(\mathcal{T}_{\sigma}^{R c}\right)$ barriers approximately

$$
\left[G_{\sigma}^{c}\right]_{\mathrm{off-res}} \propto \mathcal{T}_{\sigma}^{L c} \mathcal{T}_{\sigma}^{R c} \quad \text { and } \quad\left[G_{\sigma}^{c}\right]_{\mathrm{res}} \propto \frac{\mathcal{T}_{\sigma}^{R c}}{\mathcal{T}_{\sigma}^{L c}} .
$$

Consequently, one can see that, for resonant transport, contributions due to the spin selectivity of the barriers cancel each other out if these barriers exhibit identical properties in terms of spin-dependent transparency. Interestingly, by optimizing the barriers, one also expects to observe a positive ( $\left.\mathrm{TMR}_{\mathrm{res}}^{\mathrm{as}}\right)^{\prime}$ in the resonant transport case, which is generically negative as given by Eq. (28). This positive value can be achieved by forcing $\alpha_{L}>\alpha_{R}$, with a further constraint put on $\alpha_{L}$ determined by the value of $p$. Large positive values of $\left(\mathrm{TMR}_{\mathrm{res}}^{\mathrm{as}}\right)^{\prime}$ are especially expected for $\alpha_{L}>0$, which means that the left barrier should favor minority (spin-down) electrons. For instance, let us assume that only the left barrier is modified to be spin 
selective, that is, $\alpha_{L} \equiv \alpha$ and $\alpha_{R}=0$. We find numerically (for $p=0.25$ ) that $\left(\mathrm{TMR}_{\mathrm{res}}^{\mathrm{as}}\right)^{\prime}>0$ as soon as $\alpha>\alpha_{0}$, with $\alpha_{0} \approx 0.14$, and the increase of $\alpha$ is followed by the monotonic growth of (TMR $\left.\mathrm{TMes}_{\mathrm{res}}^{\text {as }}\right)^{\prime}$ up to a value approximately equal to $73 \%$ for $\alpha=1$ - the maximal achievable value for a given $p$. Interestingly, if one could fabricate a device with $\alpha_{L}=-\alpha_{R}=\alpha$ - that is, with the tunnel barriers of perfectly antisymmetric spin-selective propertiesdoing so would allow for achieving $\alpha_{0} \approx 0.07$ and $\left(\mathrm{TMR}_{\mathrm{res}}^{\mathrm{as}}\right)^{\prime} \gtrsim 50 \%$ already at $\alpha=0.3$.

\section{B. The case of many orbital channels}

In this section, we relax the assumption regarding the position of the Fermi level around the charge-neutrality point (i.e., $E_{F}^{w}=0$ ) and assume that the level has been shifted; see the right side of Fig. 1(b). For illustrative purposes, we consider two cases, $E_{F}^{w}=400 \mathrm{meV}$ and $E_{F}^{w}=650 \mathrm{meV}$, which means that $2(n=0,1)$ and 3 $(n=0,1,2)$ orbital channels (subbands), respectively, are available for charge and spin transport through the device.

The key difference with respect to the single-channel case stems from the fact that now conductance $G_{\mathrm{P} / \mathrm{AP}}$ [Eq. (18)] for each magnetic configuration has to be summed over all orbital transport channels. Since each channel is described by a different transmission coefficient $\mathscr{T}_{\sigma n}^{\mathrm{P} / \mathrm{AP}}$ [Eq. (19)], the characteristic energies $\tilde{\varepsilon}_{\sigma p}^{c n}$ at which the resonant tunneling of electrons occurs are uniquely associated with the subband index $n$,

$$
\begin{aligned}
\tilde{\varepsilon}_{\sigma p}^{c n}= & \Delta \varepsilon_{r} \sqrt{\left[p-\frac{1}{2 \pi}\left(\varphi_{\sigma n}^{L c}+\varphi_{\sigma n}^{R c}\right)-\frac{\ell k_{F}^{w}}{\pi}\right]^{2}+\left(\frac{\ell n}{r \pi}\right)^{2}} \\
& +E_{g}-E_{F}^{w},
\end{aligned}
$$

for $p \in \mathbb{Z}$. Consequently, resonances in conductance for channels characterized by various $n$ 's appear at different intervals, which, in turn, leads to a complex pattern of total conductance as a function of $E_{g}$. This effect is illustrated in the top panels of Fig. 8, where, as an example, the total conductance in the parallel magnetic configuration (the solid line) for (a) two and (b) three orbital channels participating in transport has been decomposed into contributions from specific channels. Furthermore, the resultant TMR no longer exhibits a clear periodic pattern: see the bottom panels of Fig. 8, where the $E_{g}$ range is purposely assumed to be the same as in Fig. 2(a) to enable an easy comparison of the results. Nevertheless, one can still distinguish three distinctive regions with respect to the barrier strength $Z$, whose origin can be explained analogously as in the single-channel case; see Sec. VA. Importantly, it should be noted that, in the limit of a large $Z$, the TMR varies between two characteristic values-TMR off-res [Eq. (26)] and $\mathrm{TMR}_{\text {res }}=\mathrm{TMR}_{\text {off-res }} / 2$ - corresponding to the offresonant and resonant electron tunneling through a CNT,
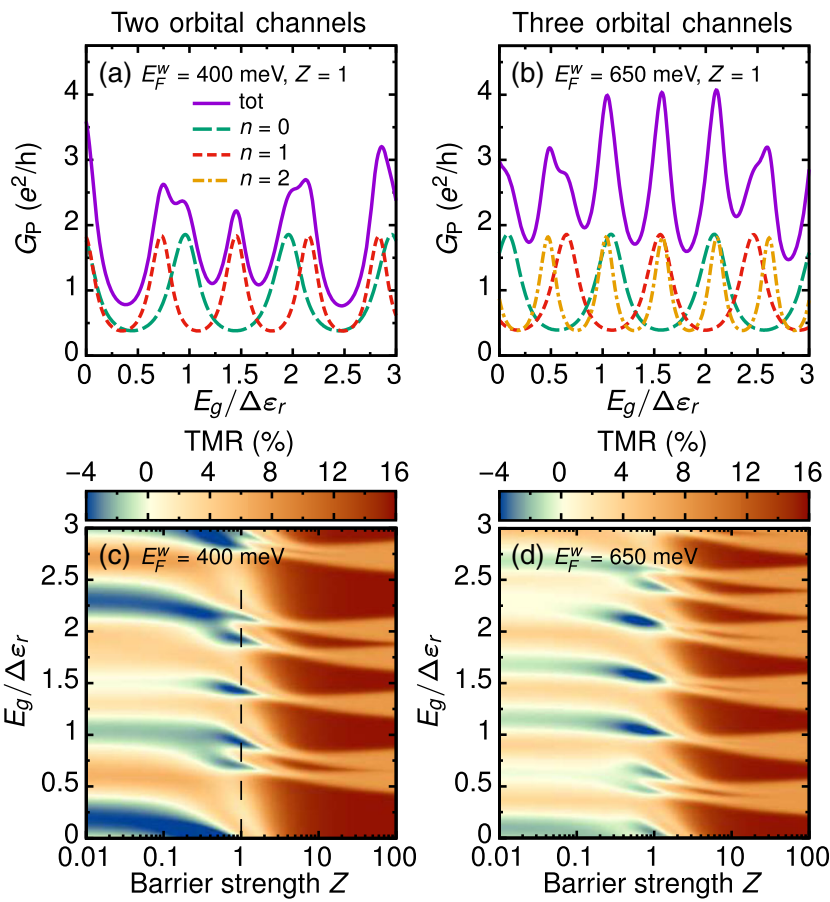

FIG. 8. The effect of many orbital channels on the transport properties of a CNT-based spin valve. The number of such channels participating in transport is modified here by assuming different shifts of the Fermi level $E_{F}^{w}$. In the left column, $E_{F}^{w}=$ $400 \mathrm{meV}$ (two orbital channels included), whereas, in the right column, $E_{F}^{w}=650 \mathrm{meV}$ (three orbital channels included). (a),(b) Conductance $G_{\mathrm{P}}$ in the parallel magnetic configuration (the solid line) decomposed into contributions from different orbital channels (the dashed and dotted-dashed lines) shown as a function of the shift of the Fermi level $E_{g}$ and $Z_{L}=Z_{R}=Z=1$. (c),(d) Density map of the tunneling magnetoresistance (TMR) plotted as a function of $E_{g}$ and the barrier strength $Z$. Note that the cross sections of (c) for $Z=1$ (that is, along the horizontal thin dashed line) and $Z=100$ are shown in Fig. 9(b). All other parameters are the same as in Fig. 2.

respectively. As the number of orbital channels participating in transport increases, the chance of resonant tunneling also becomes larger because each channel has its own unique set of resonant energies (46). As a result, one expects that, with an increasing channel number, the TMR should take a resonant value more often, as observed when comparing Figs. 8(c) and 8(d).

Next, we analyze how the asymmetry of the strength between the left and right barriers $\left(Z_{L} \neq Z_{R}\right)$ affects the TMR signal. For this purpose, we assume that the left barrier is fixed at $Z_{L}=1$ and we alter the strength of the right barrier $Z_{R}$; see Fig. 9. The cross section of Fig. 9(a) along $Z_{R}=1$ corresponds then to the cross section along the thin dashed line in Fig. 8(c), and it represents the case of symmetric barriers. For $Z_{R} \ll 1$, which represents the situation of the right barrier being almost fully transparent, one can see a softening of TMR features which is accompanied by a smearing out of some resonances; see 


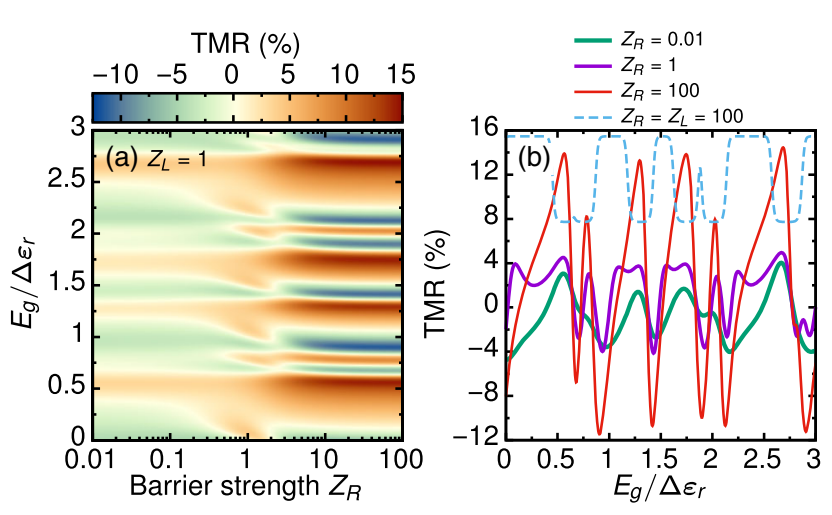

FIG. 9. Influence of the barrier-strength asymmetry on the tunneling magnetoresistance (TMR). Assuming a fixed value of the left barrier strength $Z_{L}=1$, the change of the TMR as a function of the right barrier strength $Z_{R}$ is presented in (a). Characteristic cross sections of (a) for selected values of $Z_{R}$ are shown in (b). Note that the case of $Z_{R}=1$ corresponds to symmetric barriers $\left(Z_{L}=Z_{R}\right)$, and thus it also represents the cross section of Fig. 8(c) along the horizontal thin dashed line. Moreover, the result for asymptotically large, symmetric barriers $\left(Z_{L}=Z_{R}=100\right)$ is also shown (the dashed line). Here, we assume that $E_{F}^{w}=400 \mathrm{meV}$ and two orbital channels are taken into account, whereas the remaining parameters are the same as in Fig. 2.

the relevant lines in Fig. 9(b). On the other hand, in the opposite limit $\left(Z_{R} \gg 1\right)$ - that is, for a strong asymmetry between the barriers, with the right barrier being of vanishingly small transmission-TMR features become generally much sharper, forming a sawlike pattern, and TMR values vary in a broader range. Interestingly, it can be seen that peaks and dips developing in the TMR evolve from the same features which survive also in the low $Z_{R}$ limit. In addition, an especially stark contrast between symmetric (dashed line) and asymmetric (thin solid line) tunnel barriers is seen in the $Z_{R}$ limit under consideration.

Finally, to make the present discussion complete, we also investigate the effect of spin-selective barriers. Since this aspect is extensively analyzed in Sec. VA 3 for the case of a single orbital channel, here we focus only on a specific situation with two identical barriers, that is, when $Z_{L}=$ $Z_{R}=Z$ and $\alpha_{L}=\alpha_{R}=\alpha$. In Fig. 10, we show the evolution of the TMR as a function of the spin-asymmetry parameter $\alpha$ and the shift of the Fermi level $E_{g}$ for two representative values of the barrier strength: $Z=1$ [Fig. 10(a)] and $Z=$ 100 [Fig. 10(c)], with selected cross sections for chosen values of $\alpha$ given in Figs. 10(b) and 10(d), respectively. It can be seen that additional spin filtering of electrons by tunnel barriers can substantially modify the observed TMR. In the limit of a large $Z$, illustrated in the bottom panel of Fig. 10, it can be seen that, for the off-resonance regions, marked in Fig. 10(d) as shaded areas, the TMR suffers significant changes when $\alpha$ is appreciably large, while in the resonant regions the observed variation of the TMR effect is more moderate. Moreover, in the former case, the dependence of

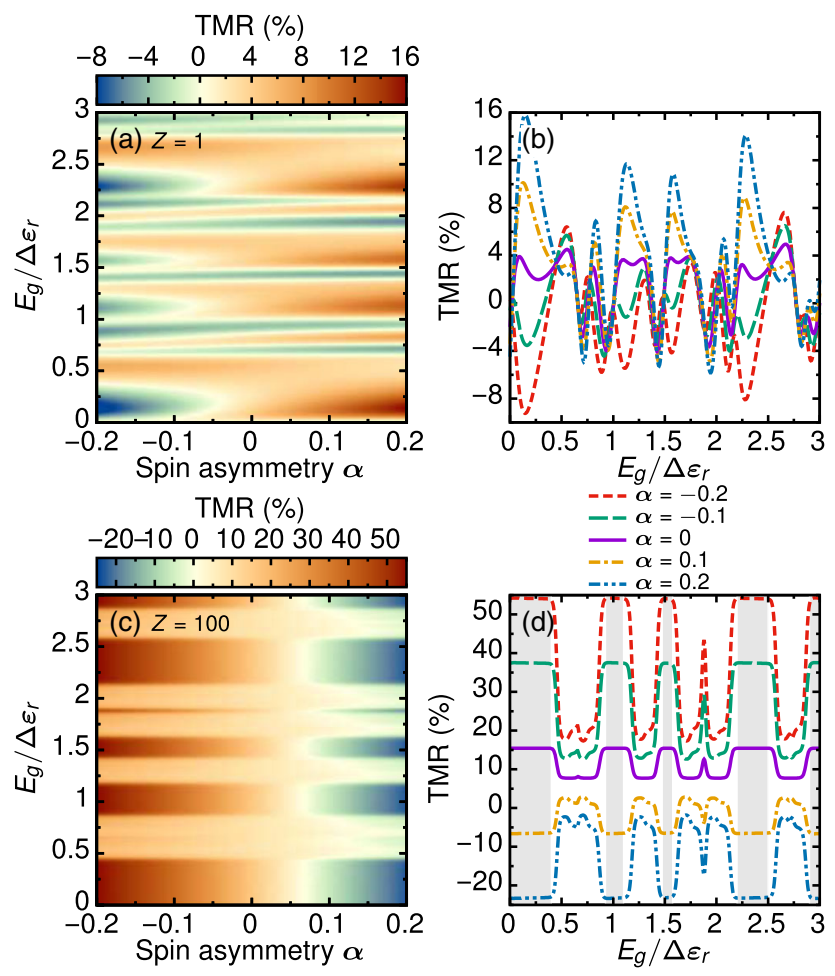

FIG. 10. The effect of spin-selective barriers on the tunneling magnetoresistance (TMR) in the case of two identical barriers characterized by the same strength $\left(Z_{L}=Z_{R}=Z\right)$ and the spinasymmetry parameter $\alpha_{L}=\alpha_{R}=\alpha$. (a),(b) The results for $Z=1$. (c),(d) The results for $Z=100$. Plots in the right column contain selected cross sections of the respective density plots from the left column. The solid lines in (b) and (d) correspond to a situation where the barriers are spin nonselective $(\alpha=0)$. Note, additionally, that the solid line in (b) [(d)] is identical to the solid line for $Z_{R}=1$ [the dashed line] in Fig. 9(b). All other parameters are the same as in Fig. 9.

the TMR on $\alpha$ is described by Eq. (29), exactly the same as with a single orbital channel.

\section{CONCLUSIONS}

With this paper, we provide a complete physical picture of the TMR effect in CNT-based spin valves. We particularly focus on the influence of the tunnel-barrier strength and the spin selectivity of the barrier on the TMR. The largest TMR signals are generally found in the strongbarrier case where the device is tuned to be off resonant with regard to the Fabry-Pérot resonances in the onedimensional wire. For a realistic CNT-based spin valve, we find a TMR signal of $15.5 \%$, a value we realized in a recent experiment [10]. In general, the off-resonant TMR is more sensitive toward changes in the barriers that the on-resonant TMR. For instance, the off-resonant TMR increases by $\Delta \mathrm{TMR}_{\text {off-res }}=40 \%$ if spin-selective barriers are added that prefer majority (spin-up) electrons from electrode, while the resonant TMR signal does not change at all. Such a 
spin-selective barrier might be implemented by spinselective insulators as EuS or EuO. However, these materials are likely to couple antiferromagnetically to the ferromagnetic leads. Therefore, using spin-selecting molecules as a barrier is believed to be more promising with regard to enhancing the TMR signal, especially since a moderate selectivity of approximately $10 \%$ already yields a strong enhancement of the TMR signal up to $37 \%$ and since doublestranded DNA has been shown to exhibit high spin-filter efficiency [68]. Using DNA as a spin filter will require a perpendicular orientation of the magnetization of the contacts. This perpendicular orientation can be implemented by the proper choice of contact material and contact shape.

As shown before, the barrier strength in CNT spin valves can be asymmetric due to fabrication, resulting in negative values of the TMR signal [26]. We find that it is, in principle, possible to correct these negative values if the barrier of the injection contact favors minority (spin-down) electrons leading to a large positive TMR of up to 50\%-70\%. In this case, adding insulation of $\mathrm{EuS}$ or $\mathrm{EuO}$ to the lead used for the spin injection will likely yield the desired result.

In the case of intermediate barrier strength-i.e., the potential energy of the barrier matches the energy of the incident electrons at the Fermi level-we show that the magnitude of the TMR has a strong response to the gate voltage, varying from $14 \%$ to $-12 \%$ within a $5-\mathrm{mV}$ gate voltage for a realistic device and without spin-selective barriers. It is important to note that this tunability of the TMR signal is effective in the absence of spin-orbit coupling, thus preserving the long spin relaxation time inherent for carbon materials. The tunability of the TMR signal is strongest for asymmetric barriers. Adding more transport channels, e.g., by working at larger gate voltages, the number of resonances increases, leading to a less periodic pattern of the TMR with gate voltage. Changes of the TMR signal with respect to barrier strength, asymmetry, and spin selectivity, however, remain qualitatively the same.

In conclusion, we show in this paper that the feasibility of modification of the tunnel barriers in a controlled way, together with the electrical tuning of a CNT, could open up the possibility of building CNT-based devices exhibiting a large TMR effect with a strong response to the gate voltage. Specifically, a prospective way to achieve this goal lies in the application of highly asymmetric and/or spin-selective tunnel barriers. This application paves the way for spintronic devices that work without spin-orbit coupling and thus preserve long spin relaxation times.

\section{ACKNOWLEDGMENTS}

The authors thank P. Mavropoulos and J. Splettstoesser for the fruitful discussions. M. M. acknowledges financial support from the Alexander von Humboldt Foundation, the Polish Ministry of Science and Higher Education through a young scientist fellowship (No. 0066/E-336/9/2014), and the Knut and Alice Wallenberg Foundation. C.M. acknowledges financial support from DFG Research Unit FOR912 and by the "Niedersächsiche Vorab" program of the Volkswagen Stiftung.

[1] S. D. Bader and S. S. P. Parkin, Spintronics, Annu. Rev. Condens. Matter Phys. 1, 71 (2010).

[2] F. Pulizzi, Spintronics (Insight issue), Nat. Mater. 11, 367 (2012).

[3] L. E. Hueso, J. M. Pruneda, V. Ferrari, G. Burnell, J. P. Valdés-Herrera, B. D. Simons, P. B. Littlewood, E. Artacho, A. Fert, and N. D. Mathur, Transformation of spin information into large elctgrical signals using carbon nanotubes, Nature (London) 445, 410 (2007).

[4] B. Dlubak, M.-B. Martin, C. Deranlot, B. Servet, S. Xavier, R. Mattana, M. Sprinkle, C. Berger, W. A. DeHeer, F. Petroff, A. Anane, P. Seneor, and A. Fert, Highly efficient spin transport in epitaxial graphene on SiC, Nat. Phys. 8, 557 (2012).

[5] W. Han, R. K. Kawakami, M. Gmitra, and J. Fabian, Graphene spintronics, Nat. Nanotechnol. 9, 794 (2014).

[6] M. H. D. Guimarães, P. J. Zomer, J. Ingla-Aynés, J. C. Brant, N. Tombros, and B. J. van Wees, Controlling Spin Relaxation in Hexagonal BN-Encapsulated Graphene with a Transverse Electric Field, Phys. Rev. Lett. 113, 086602 (2014).

[7] M. Drögeler, F. Volmer, M. Wolter, B. Terrés, K. Watanabe, T. Taniguchi, G. Güntherodt, C. Stampfer, and B. Beschoten, Nanosecond spin lifetimes in single- and fewlayer graphene-hBN heterostructures at room temperature, Nano Lett. 14, 6050 (2014).

[8] E. A. Laird, F. Pei, and L. P. Kouwenhoven, A valley-spin qubit in a carbon nanotube, Nat. Nanotechnol. 8, 565 (2013).

[9] J. J. Viennot, M. C. Dartiailh, A. Cottet, and T. Kontos, Coherent coupling of a single spin to microwave cavity photons, Science 349, 408 (2015).

[10] C. Morgan, M. Misiorny, D. Metten, S. Heedt, Th. Schäpears, C. M. Schneider, and C. Meyer, Impact of Tunnel-Barrier Strength on Magnetoresistance in Carbon Nanotubes, Phys. Rev. Applied 5, 054010 (2016).

[11] W. Han, K. Pi, K. M. McCreary, Yan Li, Jared J. I. Wong, A. G. Swartz, and R. K. Kawakami, Tunneling Spin Injection into Single Layer Graphene, Phys. Rev. Lett. 105, 167202 (2010).

[12] I. Žutić, J. Fabian, and S. Das Sarma, Spintronics: Fundamentals and applications, Rev. Mod. Phys. 76, 323 (2004).

[13] J Sinova, S Valenzuela, J. Wunderlich, C. H. Back, and T. Jungwirth, Spin Hall effects, Rev. Mod. Phys. 87, 1213 (2015).

[14] M. Gmitra, S. Konschuh, C. Ertler, C. Ambrosch-Draxl, and J. Fabian, Band-structure topologies of graphene: Spin-orbit coupling effects from first principles, Phys. Rev. B 80, 235431 (2009).

[15] A. H. Castro Neto and F. Guinea, Impurity-Induced SpinOrbit Coupling in Graphene, Phys. Rev. Lett. 103, 026804 (2009). 
[16] D. Huertas-Hernando, F. Guinea, and A. Brataas, Spin-orbit coupling in curved graphene, fullerenes, nanotubes, and nanotube caps, Phys. Rev. B 74, 155426 (2006).

[17] S. H. Jhang, M. Marganska, Y. Skourski, D. Preusche, B. Witkamp, M. Grifoni, H. van der Zant, J. Wosnitza, and C. Strunk, Spin-orbit interaction in chiral carbon nanotubes probed in pulsed magnetic fields, Phys. Rev. B 82, 041404 (2010).

[18] G. A. Steele, F. Pei, E. A. Laird, J. M. Jol, H. B. Meerwaldt, and L. P. Kouwenhoven, Large spin-orbit coupling in carbon nanotubes, Nat. Commun. 4, 1573 (2013).

[19] K. Tsukagoshi, B. W. Alphenaar, and H. Ago, Coherent transport of electron spin in a ferromagnetically contacted carbon nanotube, Nature (London) 401, 572 (1999).

[20] J.-R. Kim, H. M. So, J.-J. Kim, and J. Kim, Spin-dependent transport properties in a single-walled carbon nanotube with mesoscopic Co contacts, Phys. Rev. B 66, 233401 (2002).

[21] B. Zhao, I. Mönch, H. Vinzelberg, T. Mühl, and C. M. Schneider, Spin-coherent transport in ferromagnetically contacted carbon nanotubes, Appl. Phys. Lett. 80, 3144 (2002).

[22] A. Jensen, J. R. Hauptmann, J. Nygård, and P. E. Lindelof, Magnetoresistance in ferromagnetically contacted singlewall carbon nanotubes, Phys. Rev. B 72, 035419 (2005).

[23] S. Sahoo, T. Kontos, C. Schönenberger, and C. Sürgers, Electrical spin injection in multiwall carbon nanotubes with transparent ferromagnetic contacts, Appl. Phys. Lett. 86 (2005).

[24] H. T. Man, I. J. W. Wever, and A. F. Morpurgo, Spindependent quantum interference in single-wall carbon nanotubes with ferromagnetic contacts, Phys. Rev. B 73, 241401 (2006).

[25] W. Liang, M. Bockrath, D. Bozovic, J. H. Hafner, M. Tinkham, and H. Park, Fabry-Perot interference in a nanotube electron waveguide, Nature (London) 411, 665 (2001).

[26] S. Sahoo, T. Kontos, J. Furer, C. Hoffmann, M. Gräber, A. Cottet, and C. Schönenberger, Electric field control of spin transport, Nat. Phys. 1, 99 (2005).

[27] H. T. Man and A.F. Morpurgo, Sample-Specific and Ensemble-Averaged Magnetoconductance of Individual Single-Wall Carbon Nanotubes, Phys. Rev. Lett. 95, 026801 (2005).

[28] A. Cottet, T. Kontos, W. Belzig, C. Schönenberger, and C. Bruder, Controlling spin in an electronic interferometer with spin-active interfaces, Europhys. Lett. 74, 320 (2006).

[29] A. Cottet, T. Kontos, S. Sahoo, H. T. Man, M.-S. Choi, W. Belzig, C. Bruder, A. F. Morpurgo, and C. Schönenberger, Nanospintronics with carbon nanotubes, Semicond. Sci. Technol. 21, S78 (2006).

[30] D. Grundler, Oscillatory Spin-Filtering due to Gate Control of Spin-Dependent Interface Conductance, Phys. Rev. Lett. 86, 1058 (2001).

[31] C.-M. Hu and T. Matsuyama, Spin Injection across a Heterojunction: A Ballistic Picture, Phys. Rev. Lett. 87, 066803 (2001).

[32] C. L. Kane and E. J. Mele, Size, Shape, and Low Energy Electronic Structure of Carbon Nanotubes, Phys. Rev. Lett. 78, 1932 (1997).
[33] R. Egger and A. O. Gogolin, Correlated transport and nonfermi-liquid behavior in single-wall carbon nanotubes, Eur. Phys. J. B 3, 281 (1998).

[34] G. E. Blonder, M. Tinkham, and T. M. Klapwijk, Transition from metallic to tunneling regimes in superconducting microconstrictions: Excess current, charge imbalance, and supercurrent conversion, Phys. Rev. B 25, 4515 (1982).

[35] Y. Qi, D. Y. Xing, and J. Dong, Relation between Julliere and Slonczewski models of tunneling magnetoresistance, Phys. Rev. B 58, 2783 (1998).

[36] Spin Current, edited by S. Maekawa, S. Valenzuela, E. Saitoh, and T. Kimura, Series on Semiconductor Science and Technology Vol. 17 (Oxford University Press, Oxford, 2012).

[37] Handbook of Spin Transport and Magnetism, edited by E. Y. Tsymbal and I. Žutić (CRC Press, Boca Raton, 2012).

[38] A. De Martino and R. Egger, Rashba spin-orbit coupling and spin precession in carbon nanotubes, J. Phys. Condens. Matter 17, 5523 (2005).

[39] T. Giamarchi, Quantum Physics in One Dimension, International Series of Monographs on Physics Vol. 121 (Oxford University Press, Oxford, 2003).

[40] M. Krüger, M. R. Buitelaar, T. Nussbaumer, C. Schönenberger, and L. Forró, Electrochemical carbon nanotube field-effect transistor, Appl. Phys. Lett. 78, 1291 (2001).

[41] M. Krüger, I. Widmer, T. Nussbaumer, M. Buitelaar, and C. Schönenberger, Sensitivity of single multiwalled carbon nanotubes to the environment, New J. Phys. 5, 138 (2003).

[42] S. Heinze, J. Tersoff, R. Martel, V. Derycke, J. Appenzeller, and $\mathrm{Ph}$. Avouris, Carbon Nanotubes as Schottky Barrier Transistors, Phys. Rev. Lett. 89, 106801 (2002).

[43] L. E. F. Foa-Torres, S. Roche, and J.-C. Charlier, Introduction to Graphene-Based Nanomaterials: From Electronic Structure to Quantum Transport (Cambridge University Press, Cambridge, England, 2014).

[44] S. Zhang and P. M. Levy, Models for magnetoresistance in tunnel junctions, Eur. Phys. J. B 10, 599 (1999).

[45] P. Mavropoulos, N. Papanikolaou, and P. H. Dederichs, Korringa-Kohn-Rostoker Green-function formalism for ballistic transport, Phys. Rev. B 69, 125104 (2004).

[46] N. Nemec, D. Tománek, and G. Cuniberti, Contact Dependence of Carrier Injection in Carbon Nanotubes: An Ab Initio Study, Phys. Rev. Lett. 96, 076802 (2006).

[47] M. A. M Gijs and G. E. W. Bauer, Perpendicular giant magnetoresistance of magnetic multilayers, Adv. Phys. 46, 285 (1997).

[48] H. Kroemer and Q.-G. Zhu, On the interface connection rules for effective-mass wave functions at an abrupt heterojunction between two semiconductors with different effective mass, J. Vac. Sci. Technol. 21, 551 (1982).

[49] Q.-G. Zhu and H. Kroemer, Interface connection rules for effective-mass wave functions at an abrupt heterojunction between two different semiconductors, Phys. Rev. B 27, 3519 (1983).

[50] W. A. Harrison, Effects of matching conditions in effectivemass theory: Quantum wells, transmission, and metalinduced gap states, J. Appl. Phys. 110, 113715 (2011).

[51] S. Datta, Electronic Transport in Mesoscopic Systems (Cambridge University Press, Cambridge, England, 1997). 
[52] J. S. Moodera, J. Nassar, and G. Mathon, Spin-tunneling in ferromagnetic junctions, Annu. Rev. Mater. Sci. 29, 381 (1999).

[53] G. Schmidt, D. Ferrand, L. W. Molenkamp, A. T. Filip, and B. J. van Wees, Fundamental obstacle for electrical spin injection from a ferromagnetic metal into a diffusive semiconductor, Phys. Rev. B 62, R4790 (2000).

[54] G. Schmidt, Concepts for spin injection into semiconductors: A review, J. Phys. D 38, R107 (2005).

[55] E. I. Rashba, Theory of electrical spin injection: Tunnel contacts as a solution of the conductivity mismatch problem, Phys. Rev. B 62, R16267 (2000).

[56] A. Fert and H. Jaffres, Conditions for efficient spin injection from a ferromagnetic metal into a semiconductor, Phys. Rev. B 64, 184420 (2001).

[57] T. Valet and A. Fert, Theory of the perpendicular magnetoresistance in magnetic multilayers, Phys. Rev. B 48, 7099 (1993).

[58] A. D. Stone and P. A. Lee, Effect of Inelastic Processes on Resonant Tunneling in One Dimension, Phys. Rev. Lett. 54, 1196 (1985).

[59] Ya. M. Blanter and M. Büttiker, Shot noise in mesoscopic conductors, Phys. Rep. 336, 1 (2000).

[60] P. J. Price, Attempt frequency in tunneling, Am. J. Phys. 66, 1119 (1998).

[61] M. Julliere, Tunneling between ferromagnetic films, Phys. Lett. 54A, 225 (1975).
[62] M. Müller, G.-X. Miao, and J. S. Moodera, Exchange splitting and bias-dependent transport in $\mathrm{EuO}$ spin filter tunnel barriers, Europhys. Lett. 88, 47006 (2009).

[63] T. Nagahama, T. S. Santos, and J. S. Moodera, Enhanced Magnetotransport at High Bias in Quasimagnetic Tunnel Junctions with EuS Spin-Filter Barriers, Phys. Rev. Lett. 99, 016602 (2007).

[64] B. Göhler, V. Hamelbeck, T. Z. Markus, M. Kettner, G. F. Hanne, Z. Vager, R. Naaman, and H. Zacharias, Spin selectivity in electron transmission through self-assembled monolayers of double-stranded DNA, Science 331, 894 (2011).

[65] D. Mishra, T. Z. Markus, R. Naaman, M. Kettner, B. Göhler, H. Zacharias, N. Friedman, M. Sheves, and C. Fontanesi, Spin-dependent electron transmission through bacteriorhodopsin embedded in purple membrane, Proc. Natl. Acad. Sci. U.S.A. 110, 14872 (2013).

[66] A.-M. Guo and Q.-F. Sun, Spin-dependent electron transport in protein-like single-helical molecules, Proc. Natl. Acad. Sci. U.S.A. 111, 11658 (2014).

[67] S. D. Pappas, P. Poulopoulos, B Lewitz, A. Straub, A. Goschew, V. Kapaklis, F. Wilhelm, A. Rogalev, and P. Fumagalli, Direct evidence for significant spin-polarization of $\mathrm{EuS}$ in $\mathrm{Co} / \mathrm{EuS}$ multilayers at room temperature, Sci. Rep. 3, 1333 (2013).

[68] Z. Xie, T. Z. Markus, S. R. Cohen, Z. Vager, R. Gutierrez, and R. Naaman, Spin specific electron conduction through DNA oligomers, Nano Lett. 11, 4652 (2011). 